\title{
Small-amplitude perturbations of shape for a nearly spherical bubble in an inviscid straining flow (steady shapes and oscillatory motion)
}

\author{
By I. S. KANG AND L. G. LEAL \\ Department of Chemical Engineering, California Institute of Technology, \\ Pasadena, CA 91125, USA
}

(Received 10 April 1987 and in revised form 29 July 1987)

The method of domain perturbations is used to study the problem of a nearly spherical bubble in an inviscid, axisymmetric straining flow. Steady-state shapes and axisymmetric oscillatory motions are considered. The steady-state solutions suggest the existence of a limit point at a critical Weber number, beyond which no solution exists on the steady-state solution branch which includes the spherical equilibrium state in the absence of flow (e.g. the critical value of 1.73 is estimated from the thirdorder solution). In addition, the first-order steady-state shape exhibits a maximum radius at $\theta=\frac{1}{6} \pi$ which clearly indicates the barrel-like shape that was found earlier via numerical finite-deformation theories for higher Weber numbers. The oscillatory motion of a nearly spherical bubble is considered in two different ways. First, a small perturbation to a spherical base state is studied with the ad hoc assumption that the steady-state shape is spherical for the complete Weber-number range of interest. This analysis shows that the frequency of oscillation decreases as Weber number increases, and that a spherical bubble shape is unstable if Weber number is larger than 4.62. Secondly, the correct steady-state shape up to $O(W)$ is included to obtain a rigorous asymptotic formula for the frequency change at small Weber number. This asymptotic analysis also shows that the frequency decreases as Weber number increases; for example, in the case of the principal mode $(n=2), \omega^{2}=\omega_{0}^{2}(1-0.31 \mathrm{~W})$, where $\omega_{0}$ is the oscillation frequency of a bubble in a quiescent fluid.

\section{Introduction}

We consider the motion of a gas bubble in a uniaxial inviscid straining flow. Specifically, we use the method of domain perturbations to study small steady deformations of shape from spherical, and small-amplitude oscillatory motions of a bubble which is initially deformed slightly to a non-equilibrium shape.

Steady-state numerical solutions for the finite-amplitude deformation of a bubble in a uniaxial inviscid straining flow were obtained recently by Miksis (1981) and Ryskin \& Leal (1984). Ryskin \& Leal used a steady-state, iterative method to solve the full Navier-Stokes equations for a series of finite values of Reynolds number $R$, with $R=\infty$ included as a limiting case. They found, for each $R \geqslant 10$, that steady, converged solutions could not be obtained beyond a certain maximum Weber number $W$, which we term below the critical Weber number. Miksis considered only the inviscid limit, but was then able to employ the powerful boundary-integral method in conjunction with Newton's method to demonstrate that the point of non- 
convergence was actually a limit point in the branch of steady solutions which contains the sphere in the absence of flow (i.e. $W \equiv 0$ ).

In a companion to the present paper, Kang \& Leal (1987) used a full timedependent numerical code to again study the problem of bubble deformation in a uniaxial extensional flow at several Reynolds numbers (including $R=\infty$ ), and a range of Weber numbers near the steady-state critical values obtained by Ryskin \& Leal (1984). They found that a bubble is elongated continuously if the Weber number is larger than the steady-state critical value. They also found that a bubble with a sufficiently large initial deformation will elongate in a similar manner even for smaller, subcritical Weber numbers. Thus a steady-state solution is possible only if the Weber number is smaller than the critical value found by Ryskin \& Leal (1984), and then only if the initial shape is not too far from the steady-state solution. Finally, in the inviscid flow limit, Kang \& Leal (1987) found that an initially deformed bubble exhibited oscillatory changes of shape at Weber numbers below critical. They also found that the frequency of oscillation decreases as Weber number increases, approaching zero at Ryskin \& Leal's critical Weber number for steady solutions. However, these numerical solutions of the initial-value problem are not sufficient to understand the oscillatory motion of a bubble in a straining flow, because we can get only the lumped global behaviour of the oscillatory motion. To understand the details of oscillatory motion such as the frequency change of each mode, the mode-mode interactions, or the evolution of a low-frequency mode to a higher-frequency mode, etc., we need a complementary analytical study.

The free oscillation of drops and bubbles in a quiescent fluid has been studied extensively since Rayleigh (1879; see also Lamb 1932) first analysed the smallamplitude oscillations of an inviscid globe. These linear results have been extended to include viscous effects (Reid 1960; Miller \& Scriven 1968; Prosperetti 1977; Marston 1980), and nonlinear oscillations of a liquid drop in a quiescent fluid have also been analysed (Tsamopoulos \& Brown 1983, 1984; Natarajan \& Brown 1987). In addition, some work has been done recently on more complicated problems such as oscillatory motion of a rotating drop (Busse 1984; Annamalai, Trinh \& Wang 1985). In spite of the extensive literature on oscillating bubbles and drops, however, relatively little has been done yet to determine how the phenomena are modified in the presence of a mean motion relative to the bubble or drop. We are aware of only one paper in this direction, by Subramanyam (1969), who studied the oscillations of a drop moving in another fluid at low values of Reynolds number and Weber number. In view of the importance of the oscillatory motion problem in understanding phenomena such as acoustic noise generation in bubbly liquids, where the bubble is almost always subjected to some non-trivial mean motion of the suspending fluid, this is rather surprising, and provides one primary motivation for the present study of the oscillatory motion of a bubble in an inviscid straining flow.

In addition, however, the analysis of small-amplitude oscillations of shape can also provide an alternative to numerical computation in determining the significance of the critical Weber number for non-convergence of steady solutions that was found by Miksis (1981) and Ryskin \& Leal (1984). In particular, since the linearized equations governing small-amplitude oscillations are the same as those derived via a linear stability analysis, the Jacobian matrix for a small disturbance to the steady state becomes singular at the zero frequency (or zero eigenvalue) point of the lowestfrequency oscillating mode. Thus, if we were to use the exact steady solution as the base state, the zero-frequency point of the lowest-frequency oscillating mode should 
be the same as the limit point of the steady solution branch which includes the spherical shape as the zero-Weber-number solution, provided only that the limit point corresponds to a physical limit and not a numerical artifact. Unfortunately, The exact steady shape is not known analytically for finite Weber numbers where the deformation becomes finite. Thus, we cannot use the exact steady solution for an analysis of oscillatory disturbance modes. The best we can do is to study the asymptotic limit for small Weber numbers where the steady shape is only slightly deformed. We may anticipate, however, that the results of such a study will be at least qualitatively reasonable for $W$ up to $O(1)$, because even the steady shapes at the critical Weber number are not drastically deformed.

In this paper we therefore consider both steady-state shapes and small-amplitude oscillatory motions of a bubble in an inviscid straining flow for small $W$. For the steady state analysis, it is advantageous to choose the magnitude of the $P_{2}(\cos \theta)$ mode of deformation $\left\langle R, P_{2}\right\rangle$ as the small parameter instead of $W$. In this way, the limit point which appears on the stable solution branch at the critical $W=W_{\mathrm{c}}$ is transformed to a regular point on the solution curve, and we can determine the Weber number as a function of $\left\langle R, P_{2}\right\rangle$ for both the unstable and stable branches for $W \leqslant W_{\text {c }}$. The perturbation solution based on $W$ is then nothing but a special form of the expansion in $\left\langle R, P_{2}\right\rangle$ for the limit $W \ll 1$ on the stable branch. For the analysis of oscillatory motion, we consider two levels of approximation. In the first, we study small perturbations to a spherical base state. In the second, we analyse the first asymptotic correction for small Weber number by including the $O(W)$-contribution to the steady-state shape. From this analysis we get an asymptotic formula for the dependence of the frequency of an oscillating bubble on $W$ in a straining flow. Finally we briefly consider weak viscous effects on the oscillation about the spherical shape. To do this, we approximate the velocity field via the potential-flow solution right up to the boundary. Thus, viscous effects are included only via the viscous stress term and the pressure correction term in the normal stress condition: this is equivalent to the classical approach of Lamb (1932) who estimated the effect of viscosity for bubble oscillation in a quiescent fluid by calculating the viscous contribution to dissipation using the inviscid-flow velocity field as a first approximation for large but finite Reynolds numbers.

\section{Problem formulation}

We consider an incompressible gas bubble of volume $\frac{4}{3} \pi a^{3}$ which is undergoing small oscillations of shape in the presence of an axisymmetric, uniaxial extensional flow of a fluid with density $\rho$ and zero viscosity as sketched in figure 1 . The surface of the bubble is assumed to be characterized completely by a uniform surface tension $\gamma$. Furthermore we neglect all effects of gravity including the hydrostatic pressure variation in the fluid. Then the governing equation of motion is

$$
\nabla^{2} \phi^{\prime}=\mathbf{0}
$$

On the bubble surface the kinematic condition and the normal stress condition must be satisfied:

$$
\begin{gathered}
-\frac{1}{\left|\nabla F^{\prime}\right|} \frac{\partial F^{\prime}}{\partial t^{\prime}}=\nabla \phi^{\prime} \cdot \boldsymbol{n}, \\
G^{\prime}\left(t^{\prime}\right)+\rho \frac{\partial \phi^{\prime}}{\partial t^{\prime}}+\frac{1}{2} \rho \nabla \phi^{\prime} \cdot \nabla \phi^{\prime}=\gamma(\nabla \cdot n),
\end{gathered}
$$




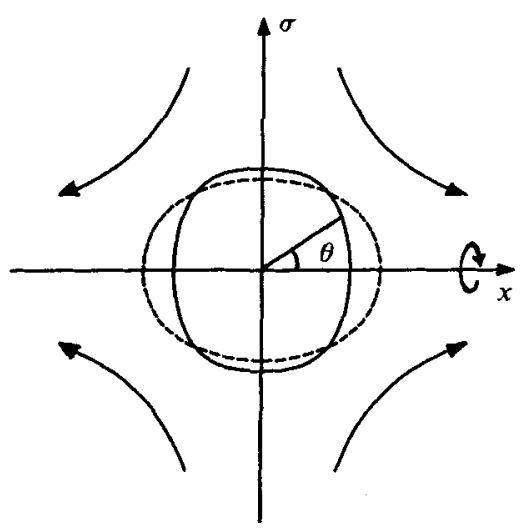

Figure 1. An oscillating bubble in an inviscid straining flow.

where $F^{\prime}$ is a function that describes the bubble shape as $F^{\prime}\left(x^{\prime}, t^{\prime}\right)=0$, and $G^{\prime}\left(t^{\prime}\right)$ is an unknown time-dependent constant which must be determined to satisfy the constraint of volume conservation. The undisturbed, uniaxial extensional flow, far from the bubble is given by

$$
\boldsymbol{u}^{\prime}=E \cdot \boldsymbol{r}^{\prime}, \quad \boldsymbol{E}=E\left(\begin{array}{rrr}
1 & 0 & 0 \\
0 & -\frac{1}{2} & 0 \\
0 & 0 & -\frac{1}{2}
\end{array}\right), \quad E>0,
$$

where $E$ is the principal strain rate. In order to non-dimensionalize the equations, we introduce characteristic velocity, length and time scales. Since all terms in the normal stress condition are equally important for oscillatory motion, the most appropriate choice is

$$
\phi_{\mathrm{c}}=\left(\frac{\gamma a}{\rho}\right)^{\frac{1}{2}}, \quad t_{\mathrm{c}}=\left(\frac{\rho a^{3}}{\gamma}\right)^{\frac{1}{2}}, \quad l_{\mathrm{c}}=a,
$$

where $a$ is the radius of the undeformed spherical bubble. Then the governing equation in dimensionless form is

$$
\nabla^{2} \phi=0,
$$

while the kinematic condition takes the form

$$
-\frac{1}{|\nabla F|} \frac{\partial F}{\partial t}=\nabla \phi \cdot n
$$

and the normal stress condition becomes

$$
G(t)+\frac{\partial \phi}{\partial t}+\frac{1}{2} \nabla \phi \cdot \nabla \phi=(\nabla \cdot n)
$$

The far-field condition (2.4), expressed in terms of the potential function $\phi$ becomes

$$
\phi_{\infty}=\left(\frac{1}{2} W\right)^{\frac{1}{2}} \frac{1}{2}\left(3 \cos ^{2} \theta-1\right)\left(\frac{1}{2} r^{2}\right),
$$

where $W$ is the Weber number, which is defined as $W=2 \rho(E a)^{2} a / \gamma$, and $\theta$ is the angle measured from the axis of symmetry. 
In addition to the differential equations and boundary conditions (2.6)-(2.9), the solution for bubble shape must satisfy two overall constraints. First, the volume of the bubble must be constant, equal to $\frac{4}{3} \pi a^{3}$, i.e.

$$
\int_{0}^{\pi} R^{3}(\theta, t) \sin \theta \mathrm{d} \theta=2
$$

where $R(\theta)$ is the unknown shape function, defined in terms of $F(x, t)$, as $F \equiv r-R(\theta$, t). Here, we have assumed that the bubble shape is axisymmetric and thus a function of the polar angle $\theta$ only. In addition to (2.10), the shape function must be defined in such a way that the centre of mass of the bubble remains at the origin. This condition can be expressed in the form

$$
\int_{0}^{\pi} R^{4}(\theta, t) \cos \theta \sin \theta \mathrm{d} \theta=0 .
$$

\section{Perturbation solution for steady-state shapes}

We consider first the steady-state problem in the limit of small deformation where the shape is nearly spherical. In this limit, it is usual to expand the solution in the form of a perturbation expansion for small $W \ll 1$, i.e.

and

$$
\begin{gathered}
\phi=\left(\frac{1}{2} W\right)^{\frac{1}{2}}\left(\phi_{0}+W \phi_{1}+W^{2} \phi_{2}+\ldots\right) \\
R=1+W \zeta_{1}+W^{2} \zeta_{2}+\ldots
\end{gathered}
$$

However, if we know, or anticipate, the existence of a limit point in the branch of stable steady solutions at some finite $W=W_{\mathrm{c}}$, it will be more convenient to pick a measure of the magnitude of deformation as an alternative small parameter $\epsilon$, and expand the solution in terms of $\epsilon$ rather than $W$. In particular, if we try to obtain an asymptotic expansion in $W$, the limit point appears as a pole in the solution which requires an infinite number of terms to resolve. However, if we expand directly in terms of an appropriate measure of the amplitude of deformation, $\epsilon$, and treat $W$ as a dependent function of $\epsilon$, the point corresponding to $W=W_{\mathrm{c}}$ becomes a regular point on the solution curve, and there is no difficulty with the approach to $W_{c}$.

For the present problem, a convenient measure of the degree of deformation, which can be used as the small parameter for an asymptotic solution, is the magnitude of the $P_{2}(\cos \theta)$ mode of deformation, i.e.

$$
\begin{aligned}
\epsilon & =\left\langle R(\theta ; \epsilon), P_{2}(\cos \theta)\right\rangle \\
& =\int_{0}^{\pi} R(\theta ; \epsilon) P_{2}(\cos \theta) \sin \theta \mathrm{d} \theta .
\end{aligned}
$$

We denote the expansion in terms of $\epsilon$ as the $P_{2}$-perturbation and the expansion in terms of $W$ as the $W$-perturbation. The $P_{2}$-perturbation takes the form

$$
\left(\begin{array}{c}
\phi \\
R \\
W
\end{array}\right)=\sum_{n=0}^{\infty} \epsilon^{n}\left(\begin{array}{c}
\left(\frac{1}{2} W\right)^{\frac{1}{2}} \tilde{\phi}_{n} \\
R_{n} \\
W_{n}
\end{array}\right),
$$

in which $W$ is treated as a function of $\epsilon$, as noted above. The expansion in terms of $\epsilon$ is equivalent to interchanging the dependent and independent variables from 

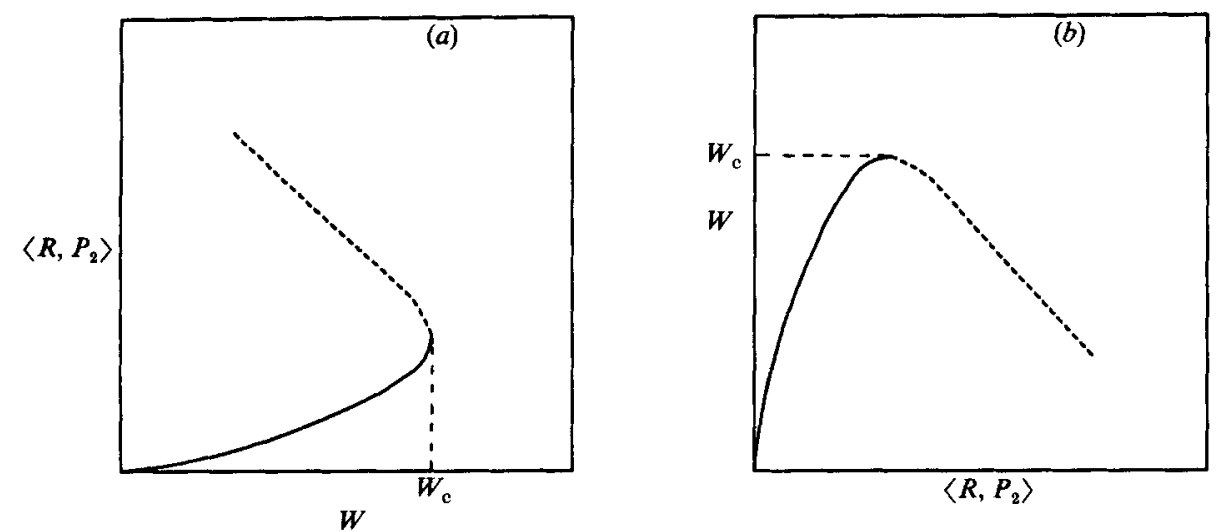

Figure 2. Representation of steady-state solutions in two different ways: $(a)\left\langle R, P_{2}\right\rangle$ in terms of $W ;(b) W$ in terms of $\left\langle R, P_{2}\right\rangle$.

$\left\langle R, P_{2}\right\rangle$ and $W$, respectively, to $W$ and $\left\langle R, P_{2}\right\rangle$. As shown in figure 2 , the solution curve for the magnitude of the $P_{2}$ mode as a function of $W$, which is singular at $W=W_{c}$, is effectively turned on its side. In the representation (3.3), the solution is regular for all $\epsilon$. Substituting (3.3) into the governing equations and boundaryconditions, we obtain

$$
\nabla^{2} \tilde{\phi}_{n}=0, \quad n=0,1,2, \ldots
$$

This equation is to be solved subject to the kinematic condition $\boldsymbol{u} \cdot \boldsymbol{n}=\boldsymbol{0}$, together with the asymptotic form (2.9) for $r \rightarrow \infty$. The bubble shape at each order in $\epsilon$ is then obtained from the normal stress balance (2.8).

To simplify the analysis, it is convenient to use the method of domain perturbations to transform the kinematic and the normal stress conditions at the bubble surface to equivalent conditions applied at $r=1$. The method is well known and we simply quote the results for the kinematic condition, which becomes

$$
\begin{aligned}
\Phi_{r 0} & +\epsilon\left[\Phi_{r 1}-\frac{\partial R_{1}}{\partial \theta} \Phi_{\theta 0}\right] \\
& +\epsilon^{2}\left[\Phi_{r 2}-\Phi_{r 1} \frac{\partial R_{1}}{\partial \theta}-\Phi_{\theta 0}\left(\frac{\partial R_{2}}{\partial \theta}-R_{1} \frac{\partial R_{1}}{\partial \theta}\right)-\frac{1}{2}\left(\frac{\partial R_{1}}{\partial \theta}\right)^{2} \Phi_{r 0}\right] \\
& +O\left(\epsilon^{3}\right)=0 \quad \text { at } r=1
\end{aligned}
$$

and the normal stress condition, which takes the form

$$
\begin{aligned}
& W_{0}\left(\Phi_{r 0}^{2}+\Phi_{\theta 0}^{2}\right) \\
& \quad+\epsilon\left[W_{1}\left(\Phi_{r 0}^{2}+\Phi_{\theta 0}^{2}\right)+W_{0}\left(2 \Phi_{r 0} \Phi_{r 1}+2 \Phi_{\theta 0} \Phi_{\theta 1}\right)\right] \\
& \quad+\epsilon^{2}\left[W_{2}\left(\Phi_{r 0}^{2}+\Phi_{\theta 0}^{2}\right)+W_{1}\left(2 \Phi_{r 0} \Phi_{r 1}+2 \Phi_{\theta 0} \Phi_{\theta 1}\right)\right. \\
& \left.\quad+W_{0}\left(\Phi_{r 1}^{2}+2 \Phi_{r 0} \Phi_{r 2}+\Phi_{\theta 1}^{2}+2 \Phi_{\theta 0} \Phi_{\theta 2}\right)\right] \\
& \quad+\epsilon^{3}\left[W_{3}\left(\Phi_{r 0}^{2}+\Phi_{\theta 0}^{2}\right)+W_{2}\left(2 \Phi_{r 0} \Phi_{r 1}+2 \Phi_{\theta 0} \Phi_{\theta 1}\right)\right. \\
& \left.\quad+W_{1}\left(\Phi_{r 1}^{2}+2 \Phi_{r 0} \Phi_{r 2}+\Phi_{\theta 1}^{2}+2 \Phi_{\theta 0} \Phi_{\theta 2}\right)+W_{0}(\ldots)\right] \\
& \quad=4(\nabla \cdot n) .
\end{aligned}
$$


In (3.5) and (3.6) the $\Phi_{i}$ are defined by

$$
\begin{aligned}
& \Phi_{r 0}=\frac{\partial \tilde{\phi}_{0}}{\partial r}, \\
& \Phi_{r 1}=\frac{\partial \tilde{\phi}_{1}}{\partial r}+R_{1}\left(\frac{\partial^{2} \tilde{\phi}_{0}}{\partial r^{2}}\right), \\
& \Phi_{r 2}=\frac{\partial \tilde{\phi}_{2}}{\partial r}+R_{1} \frac{\partial^{2} \tilde{\phi}_{1}}{\partial r^{2}}+\frac{1}{2} R_{1}^{2} \frac{\partial^{3} \tilde{\phi}_{0}}{\partial r^{3}}+R_{2} \frac{\partial^{2} \tilde{\phi}_{0}}{\partial r^{2}}, \\
& \Phi_{\theta \mathbf{0}}=\frac{\partial \tilde{\phi}_{0}}{\partial \theta}, \\
& \Phi_{\theta 1}=\frac{\partial \tilde{\phi}_{1}}{\partial \theta}+R_{1}\left(\frac{\partial^{2} \tilde{\phi}_{0}}{\partial r \partial \theta}-\frac{\partial \tilde{\phi}_{0}}{\partial \theta}\right), \\
& \Phi_{\theta 2}=\frac{\partial \tilde{\phi}_{2}}{\partial \theta}+R_{1}\left(\frac{\partial^{2} \tilde{\phi_{1}}}{\partial r \partial \theta}-\frac{\partial \tilde{\phi}_{1}}{\partial \theta}\right)+\frac{1}{2} R_{1}^{2}\left(2 \frac{\partial \tilde{\phi}_{0}}{\partial \theta}-2 \frac{\partial^{2} \tilde{\phi_{0}}}{\partial r \partial \theta}+\frac{\partial^{3} \tilde{\phi}_{0}}{\partial r^{2} \partial \theta}\right)+R_{2}\left(\frac{\partial^{2} \tilde{\phi_{0}}}{\partial r \partial \theta}-\frac{\partial \tilde{\phi}_{0}}{\partial \theta}\right) .
\end{aligned}
$$

We expand the bubble shape functions $R_{i}$ at each order in $\epsilon$ as a series of Legendre polynomials,

$$
R_{i}=\sum_{n=0}^{\infty} \beta_{n}^{(i)} P_{n}(\eta), \quad \mathrm{i}=0,1,2, \ldots
$$

where $\eta$ is defined by $\eta=\cos \theta$. Then the mean curvature which appears in the normal stress condition (3.6) can be expanded as

$$
\begin{aligned}
\boldsymbol{\nabla} \cdot \boldsymbol{n}= & 2+\epsilon \sum_{i=2}^{\infty}(i-1)(i+2) \beta_{i}^{(1)} P_{i}(\eta) \\
& +\epsilon^{2}\left[\sum_{j=0}^{\infty}(j-1)(j+2) \beta_{j}^{(2)} P_{j}-2 \sum_{i=2}^{\infty} \beta_{i}^{(1)} P_{i} \sum_{i=2}^{\infty}\left(i^{2}+i-1\right) \beta_{i}^{(1)} P_{i}\right] \\
& +\epsilon^{3}\left[\sum_{k=0}^{\infty}(k-1)(k+2) \beta_{k}^{(3)} P_{k}+3 \sum_{i=2}^{\infty} \beta_{i}^{(1)} P_{i} \sum_{i=2}^{\infty}\left(i^{2}+i-1\right) \beta_{i}^{(1)} P_{i}\right. \\
& -2\left(\sum_{i=2}^{\infty} \beta_{i}^{(1)} P_{i} \sum_{j=0}^{\infty}\left(j^{2}+j-1\right) \beta_{j}^{(2)} P_{j}+\sum_{j=0}^{\infty} \beta_{j}^{(2)} P_{j} \sum_{i=2}^{\infty}\left(i^{2}+i-1\right) \beta_{i}^{(1)} P_{i}\right) \\
& \left.+\frac{1}{2} \sum_{i=2}^{\infty} \beta_{i}^{(1)} P_{i}^{\prime} \sum_{i=2}^{\infty} \beta_{i}^{(1)}\left(1-\eta^{2}\right) P_{i}^{\prime}\left(-3 \sum_{i=2}^{\infty} i(i+1) \beta_{i}^{(1)} P_{i}+2 \sum_{i=2}^{\infty} \beta_{i}^{(1)} \eta P_{i}^{\prime}\right)\right],
\end{aligned}
$$

where $i=0$ and $i=1$ are not included to satisfy the volume conservation and the centre-of-mass condition in the $O(\epsilon)$-solution.

Finally, in addition to the boundary conditions listed above, the solution at each order in $\epsilon$ must satisfy the condition of volume conservation, which is given by

$$
\int_{-1}^{1}\left(R_{0}+\epsilon R_{1}+\epsilon^{2} R_{2}+\ldots\right)^{3} \mathrm{~d} \eta=2
$$

and the consistency condition, from the definition of $\epsilon$, which takes the form

$$
\epsilon \equiv \int_{-1}^{1}\left(R_{0}+\epsilon R_{1}+\epsilon^{2} R_{2}+\ldots\right) P_{2}(\eta) \mathrm{d} \eta
$$


In this steady-state problem, the centre-of-mass condition is automatically satisfied because of the symmetry property. Now, it is straightforward to obtain solutions up to $O\left(\epsilon^{3}\right)$, and the solution method will be briefly presented in the following section.

\subsection{Perturbation solution up to $O\left(\epsilon^{\mathbf{3}}\right)$}

\subsection{1. $O(1)$-problem}

The equations and boundary conditions for the $O(1)$-problem are

$$
\begin{gathered}
\nabla^{2} \tilde{\phi}_{0}=0 \quad(1 \leqslant r<\infty,-1 \leqslant \eta \leqslant 1), \\
\tilde{\phi}_{0} \rightarrow \frac{1}{2} P_{2}(\eta) r^{2} \quad \text { as } r \rightarrow \infty, \\
\frac{\partial \tilde{\phi}_{0}}{\partial r}=0 \quad \text { at } r=1, \\
W_{0}\left(\Phi_{r 0}^{2}+\Phi_{\theta 0}^{2}\right)+\text { const }=8 \quad \text { at } r=1, \\
\int_{-1}^{1} R_{0}^{3} \mathrm{~d} \eta=2, \\
\int_{-1}^{1} R_{0} P_{2} \mathrm{~d} \eta=0 .
\end{gathered}
$$

The solution of $(3.11 a)$ that satisfies $(3.11 b)$ and $(3.11 c)$ is

$$
\tilde{\phi}_{0}=P_{2}(\eta)\left(\frac{1}{2} r^{2}+{ }_{3}^{1} r^{-3}\right),
$$

which is the well-known potential-flow solution around the undisturbed sphere. In order to satisfy $(3.11 d)$ we have $W_{0}=0$, and from $(3.11 e)$ we get $R_{0}=1$. Then $(3.11 \mathrm{f})$, arising from the definition of $\epsilon$, is automatically satisfied at this level of approximation.

\subsection{2. $O(\varepsilon)$-problem}

Let us now turn to the $O(\epsilon)$-problem. The governing equations and boundary conditions in this case are

$$
\begin{gathered}
\nabla^{2} \tilde{\phi}_{1}=0 \quad(1 \leqslant r<\infty,-1 \leqslant \eta \leqslant 1), \\
\tilde{\phi}_{1} \rightarrow o\left(r^{2}\right) \text { as } r \rightarrow \infty, \\
\Phi_{r 1}-\frac{\partial R_{1}}{\partial \theta} \Phi_{\theta 0}=0 \quad \text { at } r=1, \\
W_{1}\left(\Phi_{r 0}^{2}+\Phi_{\theta 0}^{2}\right)=4 \sum_{i=2}^{\infty}(i-1)(i+2) \beta_{i}^{(1)} P_{i}(\eta) \text { at } r=1, \\
\int_{-1}^{1} R_{1} \mathrm{~d} \eta=0, \\
\int_{-1}^{1} R_{i} P_{2} \mathrm{~d} \eta=1 .
\end{gathered}
$$

In the normal stress condition $(3.13 d)$, we imposed the constraint of volume conservation $(3.13 e)$ and the centre-of-mass condition, i.e. $\beta_{0}^{(1)}=\beta_{1}^{(2)}=0$. 
Now, the shape function at $O(\epsilon)$ can be obtained using the normal stress condition $(3.13 d)$ and the known potential function $\tilde{\phi}_{0}$ from the $O(1)$-solution. In particular, by plugging the known solution $\tilde{\phi}_{0}$ into the normal stress condition $(3.13 d)$, we can solve for the coefficients $\beta_{n}^{(1)}$ in $R_{1}=\sum_{n=0}^{\infty} \beta_{n}^{(1)} P_{n}(\eta)$,

$$
\left.\begin{array}{l}
\beta_{2}^{(1)}=\frac{25}{672} W_{1}, \quad \beta_{4}^{(1)}=-\frac{5}{252} W_{1}, \\
\beta_{n}^{(1)}=0 \quad \text { for } n \neq 2,4 .
\end{array}\right\}
$$

Then from the constraint $(3.13 f)$, due to the definition of $\epsilon$, we get

$$
W_{1}=67.2 \text {. }
$$

Finally, we can determine the flow field at $O(\epsilon)$ by solving $(3.13 a)$ with $(3.13 b)$ and $(3.13 c)$. The solution of $(3.13 a)$ that satisfies the condition $(3.13 b)$ at infinity is given by

$$
\tilde{\phi}_{1}=\sum_{n=0}^{\infty} \alpha_{n}^{(1)} r^{-(n+1)} P_{n}(\eta)
$$

From the kinematic condition with known $R_{1}$ and $\tilde{\phi}_{0}$, we can easily determine the coefficients in this expression,

$$
\alpha_{2}^{(1)}=\frac{275}{18144} W_{1}, \quad \alpha_{4}^{(1)}=\frac{325}{11088} W_{1}, \quad \alpha_{6}^{(1)}=-\frac{125}{8316} W_{1}, \quad \alpha_{n}^{(1)}=0, \quad n \neq 2,4,6 .
$$

The constant volume constraint is automatically satisfied by $R_{1}$.

\subsection{3. $O\left(\epsilon^{2}\right)$-problem}

The equations governing the $O\left(\epsilon^{2}\right)$-problem are

$$
\begin{gathered}
\nabla^{2} \tilde{\phi}_{2}=0 \quad(1 \leqslant r<\infty,-1 \leqslant \eta \leqslant 1), \\
\tilde{\phi}_{2} \rightarrow o\left(r^{2}\right) \quad \text { as } r \rightarrow \infty, \\
\Phi_{r 2}-\Phi_{\theta 1} \frac{\partial R_{1}}{\partial \theta}-\Phi_{\theta 0}\left(\frac{\partial R_{2}}{\partial \theta}-R_{1} \frac{\partial R_{1}}{\partial \theta}\right)=0 \quad \text { at } r=1, \\
W_{2}\left(\Phi_{r 0}^{2}+\Phi_{\theta 0}^{2}\right)+W_{1}\left(2 \Phi_{r 0} \Phi_{r 1}+2 \Phi_{\theta 0} \Phi_{\theta 1}\right) \\
=4\left[\sum_{j=0}^{\infty}(j-1)(j+2) \beta_{j}^{(2)} P_{j}(\eta)-2 \sum_{i=2}^{\infty} \beta_{i}^{(1)} P_{i} \sum_{i=2}^{\infty}\left(i^{2}+i-1\right) \beta_{i}^{(1)} P_{i}\right], \\
\int_{-1}^{1}\left(R_{1}^{2}+R_{2}\right) \mathrm{d} \eta=0, \\
\int_{-1}^{1} R_{2} P_{2} \mathrm{~d} \eta=0 .
\end{gathered}
$$

Again, the shape function, $R_{2}$, at $O\left(\epsilon^{2}\right)$ can be obtained from (3.18d) using the known functions $\tilde{\phi}_{0}, \tilde{\phi}_{1}$ and the shape coefficients $\beta_{i}^{(1)}$ at $O(\epsilon)$. We find

$$
\begin{aligned}
\beta_{0}^{(2)} & =-0.321 \times 10^{-3} W_{1}^{2}, \\
\beta_{2}^{(2)} & =0, \\
\beta_{4}^{(2)} & =2.092 \times 10^{-3} W_{1}^{2}, \\
\beta_{6}^{(2)} & =-1.260 \times 10^{-3} W_{1}^{2}, \\
\beta_{8}^{(2)} & =0.467 \times 10^{-3} W_{1}^{2}, \\
\text { others } & =0 .
\end{aligned}
$$


Then, from $(3.18 f)$, we obtain

$$
W_{2}=-0.1671 W_{1}^{2}=-754.7 \text {. }
$$

Finally, the solution of (3.18a) satisfying $(3.18 b)$ is $\tilde{\phi}_{2}=\Sigma_{n=0}^{\infty} \alpha_{n}^{(2)} r^{-(n+1)} P_{n}(\eta)$ and the coefficients $\alpha_{n}^{(2)}$ are obtained from $(3.18 c)$ :

$$
\left.\begin{array}{rl}
\alpha_{0}^{(2)} & =-5.110 \times 10^{-5} W_{1}^{2}, \\
\alpha_{2}^{(2)} & =-1.025 \times 10^{-4} W_{1}^{2}, \\
\alpha_{4}^{(2)} & =1.298 \times 10^{-3} W_{1}^{2}, \\
\alpha_{6}^{(2)} & =2.019 \times 10^{-3} W_{1}^{2}, \\
\alpha_{8}^{(2)} & =-2.914 \times 10^{-3} W_{1}^{2}, \\
\alpha_{10}^{(2)} & =9.517 \times 10^{-4} W_{1}^{2}, \\
\text { others } & =0 .
\end{array}\right\}
$$

\subsection{4. $O(\epsilon)$-problem}

Since the $O\left(\epsilon^{3}\right)$-problem is extremely complicated, we calculate only $W_{3}$ from the normal stress condition and the consistency requirement from the definition of $\epsilon$. The normal stress condition is

$$
\begin{aligned}
W_{3}\left(\Phi_{r 0}^{2}+\Phi_{\theta 0}^{2}\right) & +W_{2}\left(2 \Phi_{r 0} \Phi_{r 1}+2 \Phi_{\theta 0} \Phi_{\theta 1}\right)+W_{1}\left(\Phi_{r 1}^{2}+2 \Phi_{r 0} \Phi_{r 2}+\Phi_{\theta 1}^{2}+2 \Phi_{\theta 0} \Phi_{\theta 2}\right) \\
= & 4\left[\sum_{k=0}^{\infty}(k-1)(k+2) \beta_{k}^{(3)} P_{k}+3 \sum_{i=2}^{\infty} \beta_{i}^{(1)} P_{i} \sum_{i=2}^{\infty}\left(i^{2}+i-1\right) \beta_{i}^{(1)} P_{i}\right. \\
& -2\left(\sum_{i=2}^{\infty} \beta_{i}^{(1)} P_{i} \sum_{j=0}^{\infty}\left(j^{2}+j-1\right) \beta_{j}^{(2)} P_{j}+\sum_{j=0}^{\infty} \beta_{j}^{(2)} P_{j} \sum_{i=2}^{\infty}\left(i^{2}+i-1\right) \beta_{i}^{(1)} P_{i}\right) \\
& \left.+\frac{1}{2} \sum_{i=2}^{\infty} \beta_{i}^{(1)} P_{i}^{\prime} \sum_{i=2}^{\infty} \beta_{i}^{(1)}\left(1-\eta^{2}\right) P_{i}^{\prime}\left(-3 \sum_{i=2}^{\infty} i(i+1) \beta_{i}^{(1)} P_{i}+2 \sum_{i=2}^{\infty} \beta_{i}^{(1)} \eta P_{i}^{\prime}\right)\right],
\end{aligned}
$$

and the definition of $\epsilon$ requires $\beta_{2}^{(3)}=0$. Substituting the known solutions of $\tilde{\phi}_{0}, \tilde{\phi}_{1}$, $\tilde{\phi}_{2}, R_{1}$, and $R_{2}$ into $(3.22)$, we get

$$
\beta_{2}^{(3)}=0.1488 W_{3}-0.937 \times 10^{-3} W_{1}^{3},
$$

and the condition $\beta_{2}^{(3)}=0$ then yields

$$
W_{3}=6.2970 \times 10^{-3} W_{1}^{3}=1910.9 .
$$

\subsection{Estimation of the critical Weber number}

The most important feature of the solution obtained in this subsection is that it exhibits multiple (double) steady-state solutions for Weber numbers below the critical value, but no steady-state solution beyond the critical value. Let us start with the second-order solution because we have the full solution to this accuracy. The Weber number as a function of $\epsilon$ is given by

$$
\begin{aligned}
W & =\epsilon W_{1}+\epsilon^{2} W_{2} \\
& =\left(\epsilon W_{1}\right)-0.1671\left(\epsilon W_{1}\right)^{2} \\
& =67.2 \epsilon-754.7 \epsilon^{2} .
\end{aligned}
$$


The critical Weber number occurs where $\partial W / \partial \epsilon=0$ - see figure $2(b)$. This point can thus be estimated from (3.24), which gives

$$
W_{\mathrm{c}}=1.50 \text { at } \epsilon_{\mathrm{c}}=0.0445 .
$$

This estimate of the critical Weber number 1.50 is considerably below the value 2.76 obtained numerically by Miksis (1981), but the general features of the solutions are more or less the same (see figure 3 in $\$ 3.3$ ).

The third-order solution for $W$ as a function of $\epsilon$ is

$$
\begin{aligned}
W & =\epsilon W_{1}+\epsilon^{2} W_{2}+\epsilon^{3} W_{3} \\
& =\left(\epsilon W_{1}\right)-0.1671\left(\epsilon W_{1}\right)^{2}+0.0063\left(\epsilon W_{1}\right)^{3} \\
& =67.2 \epsilon-754.7 \epsilon^{2}+1910.9 \epsilon^{3} .
\end{aligned}
$$

Equation (3.26) predicts

$$
W_{\mathrm{c}}=1.73 \text { at } \epsilon_{\mathrm{c}}=0.0568
$$

The critical Weber number 1.73 is still below the numerical value 2.76 , but comparison of the $O\left(\epsilon^{2}\right)$ - and $O\left(\epsilon^{3}\right)$-solutions indicates that the estimate of the critical Weber number may approach 2.76 as the accuracy of the perturbation solution is increased. In the later part of this section, the second- and the third-order perturbation solutions will be compared with the $W$-perturbation solution and the numerical solutions by Miksis (1981) and Ryskin \& Leal (1984).

It is interesting to note that the stability of the solution branch is exchanged at the point $\epsilon=\epsilon_{\mathrm{c}}$ (see Iooss \& Joseph 1980 for the exchange of stability at a regular turning point). To show this, we consider the following simple model equation for bubble dynamics in terms of the amplitude of the $P_{2}$-mode, $\epsilon$, which has the same steady state as the second-order solution (3.24)

$$
\frac{\mathrm{d}^{2} \epsilon}{\mathrm{d} t^{2}}=K(\epsilon ; W)\left[W-\left(67.2 \epsilon-754.7 \epsilon^{2}\right)\right]
$$

Here $K(\epsilon ; W)$ is a nonlinear function of $\epsilon$ which must be non-zero to ensure that (3.24) is the unique steady-state solution of (3.28). In fact, it is clear that $K(\epsilon ; W)$ must be a positive function since the driving force for $P_{2}$-mode deformation is just $K(\epsilon ; W)$ $W$. In (3.28), we must also note that the first-order time-derivative term is not included because viscous damping is not expected. Linearization of (3.28) about the steady-state solution gives

$$
\frac{\mathrm{d}^{2} x}{\mathrm{~d} t^{2}}+\left(67.2-1509.4 \epsilon_{s}\right) K\left(\epsilon_{\mathrm{s}} ; W\right) x=0,
$$

where $x=\epsilon-\epsilon_{\mathrm{s}}$. In (3.29) the coefficient of the second term changes sign at $\epsilon=\epsilon_{\mathrm{c}}$ from positive to negative, which clearly means that the branch for $0 \leqslant \epsilon<\epsilon_{\mathrm{c}}$ is stable (oscillatory motion) while the other branch is unstable.

\subsection{The $W$-perturbation as a limiting form of the $P_{2}$-perturbation}

One advantage of the $P_{2}$-perturbation scheme is that the $W$-perturbation solution, as shown in (3.1), is nothing but a special form of the $P_{2}$-perturbation solution for the limit $W \ll 1$ on the stable branch. Thus, we can obtain the $W$-perturbation solution very easily from the $P_{2}$-perturbation by simply taking the appropriate limiting form. The $W$-perturbation solution obtained here will be used as the base state for analysis of the oscillatory motion of a bubble. 
In the small-Weber-number limit, the solution can be expanded as

$$
\begin{gathered}
\phi=\left(\frac{1}{2} W\right)^{\frac{1}{2}}\left(\phi_{0}+W \phi_{1}+W^{2} \phi_{2}+\ldots\right), \\
R=1+W \zeta_{1}+W^{2} \zeta_{2}+\ldots
\end{gathered}
$$

(equations $(3.1 a, b))$, where

$$
\begin{gathered}
\phi_{i}=\sum_{n=0}^{\infty} \gamma_{n}^{(i)} r^{-(n+1)} P_{n}, \quad i \geqslant 1 \\
\zeta_{i}=\sum_{n=0}^{\infty} \delta_{n}^{(i)} P_{n}, \quad i \geqslant 1
\end{gathered}
$$

while in the $P_{2}$-perturbation the solution is expanded as

$$
\begin{gathered}
\phi=\left(\frac{1}{2} W\right)^{\frac{1}{2}}\left(\tilde{\phi}_{0}+\epsilon \tilde{\phi}_{1}+\epsilon^{2} \tilde{\phi}_{2}+\ldots\right), \\
R=1+\epsilon R_{1}+\epsilon^{2} R_{2}+\ldots,
\end{gathered}
$$

where

$$
\begin{gathered}
\tilde{\phi}_{i}=\sum_{n=0}^{\infty} \alpha_{n}^{(i)} r^{-(n+1)} P_{n}, \quad i \geqslant 1, \\
R_{i}=\sum_{n=0}^{\infty} \beta_{n}^{(i)} P_{n}, \quad i \geqslant 1 .
\end{gathered}
$$

From $\S 3.1$, we see that $\alpha_{n}^{(i)}=\tilde{\alpha}_{n}^{(i)} W_{1}^{i}$, and $\beta_{n}^{(i)}=\tilde{\beta}_{n}^{(i)} W_{1}^{i}$, where $\tilde{\alpha}_{n}^{(i)}, \tilde{\beta}_{n}^{(i)}$ are parameterfree constants. Thus we can write $(3.30)$ as

$$
\begin{gathered}
\phi=\left(\frac{1}{2} W\right)^{\frac{1}{2}}\left(\phi_{0}+\left(\epsilon W_{1}\right) \sum_{n=0}^{\infty} \tilde{\alpha}_{n}^{(1)} r^{-(n+1)} P_{n}+\left(\epsilon W_{1}\right)^{2} \sum_{n=0}^{\infty} \tilde{\alpha}_{n}^{(2)} r^{-(n+1)} P_{n}+\ldots\right), \\
R=\phi_{0}+\left(\epsilon W_{1}\right) \sum_{n=0}^{\infty} \tilde{\beta}_{n}^{(1)} P_{n}+\left(\epsilon W_{1}\right)^{2} \sum_{n=0}^{\infty} \tilde{\beta}_{n}^{(2)} P_{n}+\ldots
\end{gathered}
$$

Also, from the relationship between $W$ and $\left(\epsilon W_{1}\right)$ in (3.24) (i.e. for the second-order solution), we can express $\left(\epsilon W_{1}\right)$ for the stable branch in terms of $W$ :

$$
\left(\epsilon W_{1}\right)=W+0.1671 W^{2}+O\left(W^{3}\right) .
$$

Then, the $W$-perturbation solution is simply obtained by plugging (3.32) into (3.31) and collecting the same order terms in $W$.

The shape function up to $O\left(W^{2}\right)$ is given by

$$
\begin{aligned}
R=1 & +\left(\frac{25}{672} P_{2}-\frac{5}{252} P_{4}\right) W \\
& +\left(-0.321 P_{0}+6.217 P_{2}-1.223 P_{4}-1.260 P_{6}+0.467 P_{8}\right) \times 10^{-3} W^{2}+0\left(W^{3}\right) .
\end{aligned}
$$

Thus, we see that the first-order, $O(W)$, shape function is

$$
\zeta_{1}=\frac{25}{672} P_{2}(\eta)-\frac{5}{252} P_{4}(\eta)=-\frac{5}{192}\left(1-5 \eta^{2}+\frac{10}{3} \eta^{4}\right)
$$

This solution (3.34) is exactly the same $O(W)$-solution as obtained by Miksis for comparison with his numerical results. Even at this leading order of approximation, the bubble shape exhibits quite interesting results. In particular, the bubble shape is not ellipsoidal at the leading order of approximation (unlike the solution of the same problem at zero Reynolds number where $\zeta_{1}=C_{1} P_{2}(\eta)$ and the shape is 


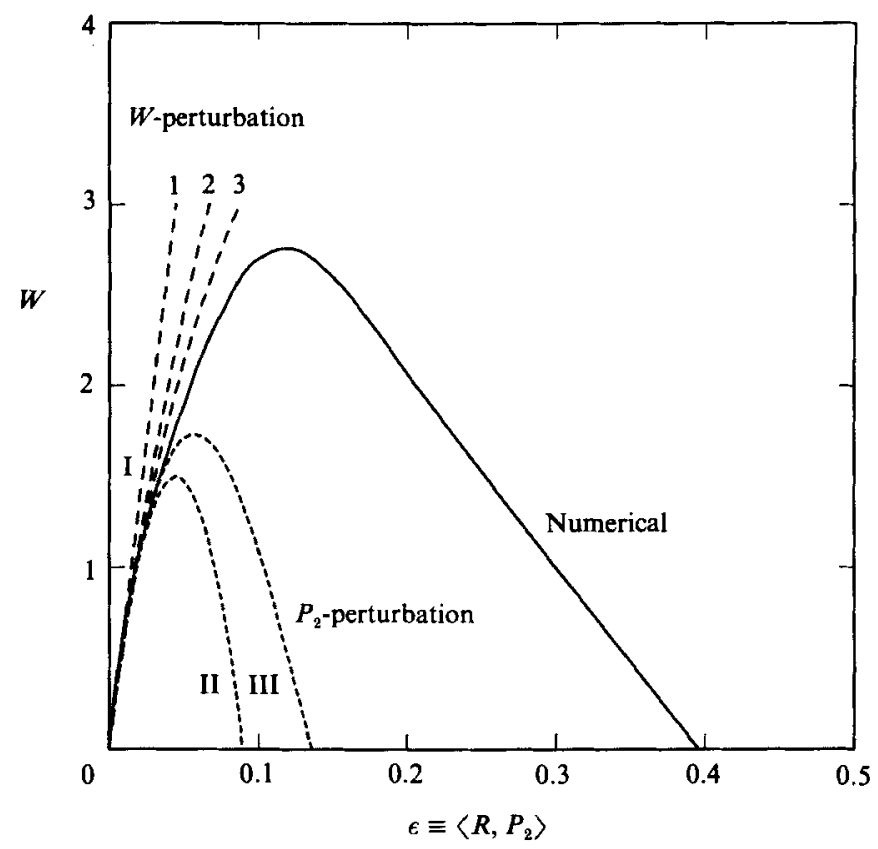

FiguRE 3. Comparison of the perturbation solutions for steady shape with the numerical analysis results of Miksis (1981) and Ryskin \& Leal (1984): - —_- 1, 2, 3, the first-, second-, and thirdorder solutions obtained by $W$-perturbation;--- I, II, III, the first-, second-, and third-order solutions obtained by the $P_{2}$-perturbation (the first-order solutions are identical in both perturbation methods); - $\longrightarrow$, numerical results.

ellipsoidal). In present case, the maximum radius does not occur at $\theta=0$ (stagnation point), but rather at

$$
\theta_{\max }=\frac{1}{6} \pi \text {. }
$$

This is a very clear first indication of the initially surprising barrel-like steady-state shape that Miksis (1981) and Ryskin \& Leal (1984) both obtained numerically. This interesting shape is obtained because the only source of deformation in this inviscid flow is the dynamic pressure variation on the bubble surface, and the dynamic pressure is clearly a maximum at the stagnation points of the flow (i.e. at the ends of the bubble and at its equator). It is also amusing to note that the maximum radius occurs at $\theta=\left(\frac{1}{2} \pi\right) / 2$ for the corresponding two-dimensional problem (Vanden-Broeck $\&$ Keller 1980), while the maximum radius occurs at $\theta=\left(\frac{1}{2} \pi\right) / 3$ for the present axisymmetric problem. The second-order solution for $W$ can be used to calculate the degree of deformation at the stagnation points, since it is these values that are usually given in experiments or numerical analysis :

$$
\left.\begin{array}{c}
f(\theta=0)=0.01736 W+0.00388 W^{2}, \\
f\left(\theta=\frac{1}{2} \pi\right)=-0.02604 W-0.00336 W^{2}
\end{array}\right\}
$$

Finally, we compare the two perturbation solutions $\left(P_{2}\right.$-perturbation and $W$ perturbation) with the numerical solutions by Miksis (1981) and Ryskin \& Leal (1984) in figure 3. Although the critical Weber number predicted by the $P_{2^{-}}$ perturbation solution is substantially below the value obtained numerically, we can see that the solution behaviour predicted by the $P_{2}$-perturbation scheme is more or 
less the same as the exact numerical solutions. Furthermore, the perturbation solution appears to approach the numerical results as the accuracy of approximation is increased. The $W$-perturbation gives a reasonable estimate of the stable portion of the solution branch, but cannot expose the limit point, which appears as a singular point, and thus would require an infinite number of terms to resolve.

\section{Small-amplitude oscillation about the steady-state shapes}

In this section, we study small-amplitude oscillations of a bubble in an inviscid, uniaxial straining flow. We are especially interested in the frequency of oscillation, which our recent numerical studies indicate as changing owing to the straining flow. Since the steady-state bubble shape is a function of Weber number, a completely rigorous analysis would require us to study the unsteady behaviour of a small disturbance from the exact steady-state shape for any specific Weber number. However, in spite of the fact that steady solutions can be obtained to any desired order in $W$, such a rigorous study would be too complicated to be tractable. Here, we approach the problem at two different levels of approximation. First, we study the unsteady dynamics of small disturbances from a spherical shape. Strictly speaking, the spherical shape represents only the limit of the steady-state solution for zero Weber number. However, Ryskin \& Leal's numerical calculations indicate that the deviation from sphericity is, in fact, relatively small $\left(f_{\mathrm{s}, \max }<0.2\right)$ even at the critical point $\left(2.7<W_{\mathrm{c}}<2.8\right)$. Hence, we may anticipate that results for a sphere will be at least qualitatively correct even for Weber numbers of $O(1)$. As a partial check on this assumption, we obtain a second solution based upon the correct steady-state shape up to $O(W)$. The latter analysis yields a first-order asymptotic formula for the frequency change as a function of Weber number.

\subsection{Oscillatory motion of a bubble about the spherical shape}

We begin with the complete equations and boundary conditions governing an axisymmetric time-dependent variation of bubble shape in an inviscid fluid. These are the equation of motion

$$
\nabla^{2} \phi=0
$$

the kinematic condition

$$
-\frac{\partial F}{\partial t}=\nabla \phi \cdot \nabla F \quad \text { at } r=1+f(\theta, t)
$$

where the bubble surface is specified by the shape function $F=r-(1+f(\theta, t))=0$, and the normal stress condition

$$
G(t)+\frac{\partial \phi}{\partial t}+\frac{1}{2} \nabla \phi \cdot \nabla \phi=(\boldsymbol{\nabla} \cdot \boldsymbol{n}) \quad \text { at } r=1+f(\theta, t) .
$$

In this first subsection, we consider small disturbances of shape for a spherical bubble in a uniaxial straining flow. The motivation for this approximate analysis was given above. The inherent assumption is that the oscillations of a sphere in a flow should reflect at least the qualitative behaviour of a real deformed bubble in the same flow. A partial check on this assumption is provided by comparing the results obtained here with the asymptotic results of $\$ 4.2$, in which we include the small $O(W)$-deformation in the steady-state bubble shape. Physically, the analysis of a 
spherical bubble will provide qualitatively correct results if the primary influence of the external flow is a direct consequence of hydrodynamic interaction between that flow and the fluctuating modes, rather than an indirect consequence of the modifications of bubble shape that are induced by the mean flow.

Let us then express the limiting forms of (4.1)-(4.3) that apply for a small disturbance from a spherical bubble shape. To do this, we introduce a small disturbance into the expression for the velocity potential for a spherical bubble, i.e.

$$
\phi=\left(\frac{1}{2} W\right)^{\frac{1}{2}} \phi_{\mathrm{s}}+\epsilon \phi_{\mathrm{u}},
$$

where

$$
\phi_{\mathrm{s}}=\frac{1}{2}\left(3 \cos ^{2} \theta-1\right)\left(\frac{1}{2} r^{2}+\frac{1}{3} r^{-3}\right)=P_{2}(\eta)\left(\frac{1}{2} r^{2}+\frac{1}{3} r^{-3}\right),
$$

and $\eta=\cos \theta$. Similarly, the shape of the bubble surface is assumed to take the form

$$
R=1+f=1+\epsilon \zeta_{\mathrm{u}}=1+\epsilon \sum_{n=2}^{\infty} \delta_{n}(t) P_{n}(\eta)
$$

Then, to obtain equations governing $\phi_{\mathrm{u}}$ and $\zeta_{\mathrm{u}}$, we substitute (4.4) and (4.6) into (4.1)-(4.3), and retain terms of $O(\epsilon)$. To obtain self-consistent results at any non-zero Weber number, $W$, with the sphere as a base case, it is necessary to neglect steady-state terms in (4.2) and (4.3) which would otherwise superpose a deterministic evolution of the base shape toward the correct equilibrium shape at any $W$. On this basis, the governing equations for small, time-dependent oscillations of bubble shape for a spherical bubble are

$$
\nabla^{2} \phi_{\mathrm{u}}=0
$$

with the kinematic condition

$$
\frac{\partial \zeta_{\mathrm{u}}}{\partial t}=\frac{\partial \phi_{\mathrm{u}}}{\partial r}+\left(\frac{1}{2} W\right)^{\frac{1}{2}} \zeta_{\mathrm{u}} \frac{\partial^{2} \phi_{\mathrm{s}}}{\partial r^{2}}-\left(\frac{1}{2} W\right)^{\frac{1}{2}} \frac{\partial \zeta_{\mathrm{u}}}{\partial \theta} \frac{\partial \phi_{\mathrm{s}}}{\partial \theta} \quad \text { at } r=1
$$

and the normal stress condition

$$
\frac{\partial \phi_{\mathrm{u}}}{\partial t}+\left(\frac{1}{2} W\right)^{\frac{1}{2}}\left(\nabla \phi_{\mathrm{s}} \cdot \boldsymbol{\nabla} \phi_{\mathrm{u}}\right)+\frac{1}{2}\left(\frac{1}{2} W\right) \zeta_{\mathrm{u}} \frac{\partial}{\partial r}\left(\nabla \phi_{\mathrm{s}} \cdot \boldsymbol{\nabla} \phi_{\mathrm{s}}\right)=\sum_{n=2}^{\infty}(n-1)(n+2) \delta_{n} P_{n}(\eta) \quad \text { at } r=1 \text {, }
$$

where $\eta=\cos \theta$. In deriving (4.8) and (4.9), the domain perturbation technique was used to transform the boundary conditions at $r=1+\epsilon \zeta_{\mathrm{u}}$ to the equivalent boundary conditions at $r=1$.

Since the solution of (4.7) that satisfies the far-field velocity condition, $\nabla \phi_{u} \rightarrow 0$ as $r \rightarrow \infty$, is given by

$$
\phi_{\mathrm{u}}=\sum_{n=0}^{\infty} \gamma_{n}(t) r^{-(n+1)} P_{n}(\eta)
$$

and we assume $\zeta_{\mathrm{u}}$ to be expressed in the form given in (4.6), the conditions (4.8) and (4.9) lead directly to dynamical equations for $\delta_{n}(t)$ and $\gamma_{n}(t)$,

$$
\begin{aligned}
\dot{\delta}_{n}= & (n+1) \gamma_{n} \\
& -\xi\left(\frac{n(n+1)(n+2)}{(2 n+3)(2 n+5)} \delta_{n+2}-\frac{n(n+1)}{(2 n-1)(2 n+3)} \delta_{n}-\frac{(n-1) n(n+1)}{(2 n-3)(2 n-1)} \delta_{n-2}\right)
\end{aligned}
$$


and

$$
\begin{aligned}
\dot{\gamma}_{n}= & (n-1)(n+2) \delta_{n} \\
& -\xi\left(\frac{(n+1)(n+2)(n+3)}{(2 n+3)(2 n+5)} \gamma_{n+2}+\frac{n(n+1)}{(2 n-1)(2 n+3)} \gamma_{n}-\frac{(n-2)(n-1) n}{(2 n-3)(2 n-1)} \gamma_{n-2}\right) \\
& -\xi^{2}\left(I_{+4}(n) \delta_{n+4}+I_{n+2}(n) \delta_{n+2}+I_{+0}(n) \delta_{n}+I_{-2}(n) \delta_{n-2}+I_{-4}(n) \delta_{n-4}\right),
\end{aligned}
$$

where $\xi=\frac{5}{2}\left(\frac{1}{2} W\right)^{\frac{1}{2}}$ and the $I_{i}(n)$ are given by

$$
\begin{aligned}
& I_{+4}(n)=A(n+4) A(n+2), \\
& I_{+2}(n)=A(n+2) B(n)+A(n+2) B(n+2)-A(n+2), \\
& I_{+0}(n)=A(n) C(n-2)+B(n) B(n)+C(n) A(n+2)-B(n), \\
& I_{-2}(n)=C(n-2) B(n-2)+C(n-2) B(n)-C(n-2), \\
& I_{-4}(n)=C(n-4) C(n-2),
\end{aligned}
$$

with

$$
\begin{aligned}
& A(n)=\frac{n(n-1)}{(2 n-1)(2 n+1)}, \\
& B(n)=\frac{2 n^{2}+2 n-1}{(2 n-1)(2 n+3)}, \\
& C(n)=\frac{(n+1)(n+2)}{(2 n+1)(2 n+3)} .
\end{aligned}
$$

In the derivation of (4.11) and (4.12), we have used the well-known recurrence formulae for Legendre polynomials

$$
\begin{gathered}
\left(1-\eta^{2}\right) P_{n}^{\prime}(\eta)=\frac{n(n+1)}{2 n+1}\left(P_{n-1}(\eta)-P_{n+1}(\eta)\right), \\
\eta P_{n}(\eta)=\frac{n}{2 n+1} P_{n-1}+\frac{n+1}{2 n+1} P_{n+1}, \\
\eta^{2} P_{n}(\eta)=\frac{n(n-1)}{(2 n-1)(2 n+1)} P_{n-2}+\frac{2 n^{2}+2 n-1}{(2 n-1)(2 n+3)} P_{n}+\frac{(n+1)(n+2)}{(2 n+1)(2 n+3)} P_{n+2} \cdot
\end{gathered}
$$

By eliminating $\gamma_{n}$ from (4.11) and (4.12), we obtain an equation for the amplitude of the shape coefficients, $\delta_{n}(t)$,

$$
\begin{aligned}
\ddot{\delta}_{n}+ & \left(\left(n^{2}-1\right)(n+2)-\xi^{2} G_{0}(n)\right) \delta_{n} \\
= & -\xi\left(J_{+2}(n) \dot{\delta}_{n+2}-J_{-2}(n) \dot{\delta}_{n-2}\right) \\
& \quad-\xi^{2}\left(G_{+4}(n) \delta_{n+4}-G_{+2}(n) \delta_{n+2}-G_{-2}(n) \delta_{n-2}+G_{-4}(n) \delta_{n-4}\right),
\end{aligned}
$$

where

$$
\begin{aligned}
& J_{+2}(n)=\frac{(n+1)(n+2)(2 n+1)}{(2 n+3)(2 n+5)}, \\
& J_{-2}(n)=\frac{n(n+1)}{2 n-1},
\end{aligned}
$$




$$
\begin{aligned}
& G_{+4}(n)=(n+1)\left(H_{+4}(n)-I_{+4}(n)\right), \\
& G_{+2}(n)=(n+1)\left(H_{+2}(n)+I_{+2}(n)\right), \\
& G_{+0}(n)=(n+1)\left(H_{+0}(n)+I_{+0}(n)\right), \\
& G_{-2}(n)=(n+1)\left(H_{-2}(n)+I_{-2}(n)\right), \\
& G_{-4}(n)=(n+1)\left(H_{-4}(n)-I_{-4}(n)\right),
\end{aligned}
$$

and

$$
\begin{aligned}
& H_{+4}(n)=\frac{(n+1)(n+2)^{2}(n+3)(n+4)}{(2 n+3)(2 n+5)(2 n+7)(2 n+9)} \\
& H_{+2}(n)=\frac{(n+1)(n+2)^{2}(n+3)}{(2 n+3)^{2}(2 n+5)(2 n+7)}-\frac{n^{2}(n+1)(n+2)}{(2 n-1)(2 n+3)^{2}(2 n+5)}, \\
& H_{+0}(n)=\frac{(n+1)^{2}(n+2)^{2}(n+3)}{(2 n+1)(2 n+3)^{2}(2 n+5)}+\frac{n^{2}(n+1)}{(2 n-1)^{2}(2 n+3)^{2}}+\frac{(n-2)^{2}(n-1) n^{2}}{(2 n-1)^{2}(2 n-3)(2 n+1)}, \\
& H_{-2}(n)=\frac{(n-1) n^{2}(n+1)}{(2 n-3)(2 n-1)^{2}(2 n+3)}-\frac{(n-2)^{2}(n-1) n}{(2 n-5)(2 n-3)(2 n-1)^{2}}, \\
& H_{-4}(n)=\frac{n(n-1)(n-2)^{2}(n-3)}{(2 n-1)(2 n-3)(2 n-5)(2 n-7)} .
\end{aligned}
$$

From (4.14), we note that there is no $\dot{\delta}_{n}$ term (i.e. no damping, as expected in an analysis which neglects all viscous effects), but there is a form of mode-mode interaction in which even modes interact only with other even-mode oscillations (or odd modes interact only with odd modes). In the absence of the mean flow (i.e. for $\xi=0$ ), interactions between modes only occur at higher order in the amplitude parameter $\epsilon$.

Since (4.14) is a system of highly coupled equations, it is advantageous to begin by studying the asymptotic behaviour for several limiting cases. First, we study the asymptotic form for the limit $n \rightarrow \infty$, and then the asymptotic form for very small values of the Weber number. Following this, we study the behaviour of (4.14) for a wide range of Weber numbers by evaluating the eigenvalues numerically.

\subsubsection{Asymptotic form for the limit $n \rightarrow \infty$}

Equation (4.14) reduces to a very simple asymptotic form in the limit $n \rightarrow \infty$. If we rescale time as $\tilde{t}=\omega t$, then

$$
\omega^{2} \frac{\mathrm{d}^{2} \delta_{n}}{\mathrm{~d} \tilde{t}^{2}}+\left(\left(n^{2}-1\right)(n+2)\right) \delta_{n}=-\xi\left(J_{+2}(n) \omega \frac{\mathrm{d} \delta_{n+2}}{\mathrm{~d} \tilde{t}}-J_{-2}(n) \omega \frac{\mathrm{d} \delta_{n-2}}{\mathrm{~d} \tilde{t}}\right)-\xi^{2}\left(O\left(n^{2}\right)\right) .
$$

Thus, if we take $\omega^{2}=\left(n^{2}-1\right)(n+2)$, it is clear that the right-hand side will be $O\left(n^{\frac{5}{2}}\right)$ because the $J_{i}$ are $O(n)$, and (4.14) is reduced to

$$
\frac{\mathrm{d}^{2} \delta_{n}}{\mathrm{~d} \tilde{t}^{2}}+\delta_{n}=0 \quad \text { as } n \rightarrow \infty
$$

The physical significance of this result is that the high-frequency modes undergo pure oscillation with no mode-mode interactions. Therefore if there is no high-frequency 
oscillation initially, then there will be no induced high-frequency oscillation. It may also be noted that the oscillation frequency, in this limit, is identical with the oscillation frequency of a bubble in the absence of any bulk flow.

\subsubsection{Asymptotic form for very small Weber number $\left(O\left(W^{\frac{1}{2}}\right)\right)$}

As shown in the analysis of $\S 3$, the steady-state bubble shape is spherical up to $O\left(W^{\frac{1}{2}}\right)$. Therefore the unsteady motion represented by (4.14) is asymptotically rigorous only for very small values of Weber number. In this subsection, we analyse this limit by retaining terms up to $O(\xi)$ from $(4.14)\left(\xi=\frac{5}{2}\left(\frac{1}{2} W\right)^{\frac{1}{2}}\right)$. The result is

$$
\ddot{\delta}_{n}+\left(\left(n^{2}-1\right)(n+2)\right) \delta_{n}=-\xi_{+2}(n) \dot{\delta}_{n+2}+\xi J_{-2}(n) \dot{\delta}_{n-2} \text { for } n \geqslant 2
$$

In fact (4.15) is a system of infinitely many coupled equations. However, because we know that there is uncoupling of the modes in the limit $n \rightarrow \infty$, it is sufficient to study a truncated system containing an arbitrary, but finite number of coupled equations, say $N$ equations.

We can easily demonstrate, for arbitrarily large (but finite) $N$, that the real parts of the eigenvalues are always zero, which means that the bubble motion is purely oscillatory in this small- $W$ limit. In order to do this, it is convenient to rewrite (4.15) in the form

$$
\ddot{\delta}_{n}+K_{n} \delta_{n}=-L_{n} \dot{\delta}_{n+2}+M_{n} \dot{\delta}_{n-2},
$$

where $n \leqslant 2 N$ (an arbitrary, but finite number), and $K_{n}, L_{n}$, and $M_{n}$ are positive functions of $n$. Then, changing variables as

$$
x_{n-1}=\delta_{n}, \quad x_{n}=\dot{\delta}_{n},
$$

(4.16) can be expressed (e.g. for the even-mode oscillations) in terms of the coupled set

$$
\left.\begin{array}{c}
\dot{x}_{n-1}=x_{n}, \\
\dot{x}_{n}=-K_{n} x_{n-1}-L_{n} x_{n+2}+M_{n} x_{n-2}
\end{array}\right\} \text { for } n=2,4, \ldots, 2 N .
$$

In vector form, this can be written as

$$
\dot{\boldsymbol{x}}=\boldsymbol{A} \cdot \boldsymbol{x}
$$

where $\boldsymbol{A}$ is

$$
\boldsymbol{A}=\left(\begin{array}{rrrrrrrrr}
0 & 1 & & & & & & & \\
-K_{2} & 0 & 0 & -L_{2} & & & & & \\
& 0 & 0 & 1 & & & & & \\
& M_{4} & -K_{4} & 0 & 0 & -L_{4} & & & \\
& & & 0 & 0 & 1 & & & \\
& & & M_{6} & -K_{6} & 0 & 0 & -L_{6} & \\
& & & & & & \ddots & & \\
& & & & & & 0 & 0 & 1 \\
& & & & & & M_{2 N} & -K_{2 N} & 0
\end{array}\right)
$$

This coefficient matrix has the generalized antisymmetric property

$$
a_{i j}=-c(i, j) a_{j i},
$$


where $c(i, j)$ is a constant whose value depends on $i$ and $j$ (it is important to note that there is no summation intended in this equation). It is easy to show that a diagonal matrix $\boldsymbol{S}$ exists which transforms a matrix of the form $\boldsymbol{A}$ in (4.17) into a true antisymmetric matrix $B$, i.e.

$$
B=S^{-1} A S
$$

where

$$
b_{i j}=-b_{j i}
$$

Moreover, the matrices $\boldsymbol{A}$ and $\boldsymbol{B}$ have the same eigenvalues. But a general property of an antisymmetric matrix is that all its eigenvalues are purely imaginary. Thus, it follows that the system of equations (4.15), for arbitrarily large but finite $N$, has only purely imaginary eigenvalues, and the motion of a bubble for $W \ll 1$ will be strictly oscillatory.

Although a general solution of (4.15) does not appear at first to be possible because of mode coupling, it is instructive to examine the truncated system for $2 N=4$ in which we consider the interaction between only the $n=2$ and $n=4$ modes. Solving (4.15) for $2 N=4$, we find

$$
-\lambda_{2}^{2}=\omega_{2}^{2}=12+O\left(\xi^{2}\right), \quad-\lambda_{4}^{2}=\omega_{4}^{2}=90+O\left(\xi^{2}\right) .
$$

Thus, mode interaction is negligible up to $O(\xi)$ (or $O\left(W^{\frac{1}{2}}\right)$ ). Although we have explicitly considered only the interaction between $n=2$ and $n=4$, this result can be generalized for arbitrary $n$ and $N$. The general treatment will be given in $\$ 4.2$ following (4.34). From this argument, we can see that the frequency of oscillation does not change due to the straining flow up to this order, i.e. there is no $O\left(W^{\frac{1}{2}}\right)$-term in the axisymmetric expression for the frequency change as a function of Weber number.

\subsubsection{Numerical evaluation of eigenvalues for larger Weber number}

In deriving (4.14) no restriction was imposed on Weber number except indirectly via the assumption that the steady shape is spherical. In the present subsection, we adopt the ad hoc point of view that was discussed at the beginning of this section, and numerically evaluate the eigenvalues of (4.14) for the whole range of Weber numbers up to values of $O(1)$, in order to study the influence of the bulk flow on shape oscillations for a spherical bubble. As discussed earlier, we presume that this will reflect at least qualitatively the behaviour of a real (deformed) bubble in the same situation.

We begin by rewriting (4.14) in the form

$$
\ddot{\delta}_{n}+\tilde{K}_{n} \delta_{n}=-L_{n} \dot{\delta}_{n+2}+M_{n} \dot{\delta}_{n-2}-S_{n} \dot{\delta}_{n+4}+T_{n} \delta_{n+2}+U_{n} \delta_{n-2}-V_{n} \delta_{n-4}
$$

Thus, defining $x_{n-1}=\delta_{n}, x_{n}=\dot{\delta}_{n}$ (e.g. for even modes) again, we find

$$
\begin{gathered}
\dot{x}_{n-1}=x_{n}, \\
\dot{x}_{n}=-\tilde{K}_{n} x_{n-1}-L_{n} x_{n+2}+M_{n} x_{n-2}-S_{n} x_{n+3}+T_{n} x_{n+1}+U_{n} x_{n-3}-V_{n} x_{n-5} \\
\text { for } n=2,4, \ldots, 2 N .
\end{gathered}
$$

In vector form $\quad \dot{x}=A \cdot x$, 


$\begin{array}{lccccc}\searrow 2 N & & & & & \\ W \backslash & 2 & 4 & 6 & 10 & 20 \\ 0 & 3.464 \mathrm{i} & 3.464 \mathrm{i} & 3.464 \mathrm{i} & 3.464 \mathrm{i} & 3.464 \mathrm{i} \\ 0.1 & 3.429 \mathrm{i} & 3.410 \mathrm{i} & 3.410 \mathrm{i} & 3.410 \mathrm{i} & 3.410 \mathrm{i} \\ 1.0 & 3.095 \mathrm{i} & 2.932 \mathrm{i} & 2.921 \mathrm{i} & 2.921 \mathrm{i} & 2.921 \mathrm{i} \\ 2.0 & 2.675 \mathrm{i} & 2.401 \mathrm{i} & 2.360 \mathrm{i} & 2.358 \mathrm{i} & 2.358 \mathrm{i} \\ 3.0 & 2.175 \mathrm{i} & 1.847 \mathrm{i} & 1.757 \mathrm{i} & 1.752 \mathrm{i} & 1.752 \mathrm{i} \\ 4.0 & 1.519 \mathrm{i} & 1.214 \mathrm{i} & 1.027 \mathrm{i} & 1.017 \mathrm{i} & 1.017 \mathrm{i} \\ 4.618 & \ldots & \ldots & \ldots & \ldots & 0 \\ 5.0 & 0.343 & 0.302 & 0.750 & 0.745 & 0.745 \\ 6.0 & 1.594 & 1.144 & 1.338 & 1.307 & 1.306\end{array}$

TABLE 1. Eigenvalue of $n=2$ mode as a function of Weber number and the number of coupled equations

where

$$
\boldsymbol{A}=\left(\begin{array}{ccccccccc}
0 & 1 & & & & & & & \\
-\tilde{K}_{2} & 0 & T_{2} & -L_{2} & -S_{2} & & & & \\
0 & 0 & 0 & 1 & 0 & & & & \\
U_{4} & M_{4} & -\tilde{K}_{4} & 0 & T_{4} & -L_{4} & -S_{4} & & \\
0 & 0 & 0 & 0 & 0 & 1 & 0 & & \\
-V_{6} & 0 & U_{6} & M_{6} & -\tilde{K}_{6} & 0 & T_{6} & -L_{6} & \\
& & & & & & \ddots & & \\
& & & 0 & 0 & 0 & 0 & 0 & 1 \\
& & & -V_{2 N} & 0 & U_{2 N} & M_{2 N} & -\tilde{K}_{2 N} & 0
\end{array}\right)
$$

The eigenvalues of $\boldsymbol{A}$ were calculated numerically for several Weber numbers by varying $N$ until converged values were obtained for the particular mode, $n$, of interest. From this numerical calculation, we found that the eigenvalues can be either purely real or purely imaginary. For example, the eigenvalues of the $n=2$ mode for several Weber numbers and different levels of truncation (i.e. values of $N$ ) are shown in table 1 . As we can see, the calculated eigenvalues for the $n=2$ mode show very good convergence as $N$ increases. With a large number $N$ included, the critical Weber number, at which the square of the $n=2$ mode eigenvalue changes sign from negative to positive, was found to be 4.62. If we neglect any intermode interaction (i.e. consider only $2 N=n=2$ corresponding to the first column in table 1 ), we get the crudest estimation of the critical Weber number as 4.95 . Since this crudest estimation overestimates the final critical value of 4.62 by only $7 \%$, it is obvious that intermode interaction does not have a strong effect on the results.

In figure 4 , the eigenvalues for both the $n=2$ and $n=3$ modes (estimated for $2 N=20$ ) are shown as a function of Weber number. The upper graph shows that both eigenvalues change from pure imaginary to pure real at critical Weber numbers ( 4.62 for $n=2$ mode and 8.58 for $n=3$ mode). The lower graph shows the real parts of these eigenvalues as a function of Weber number. Since the magnitude of the imaginary eigenvalue decreases with increase of $W$, for small $W$, we can see that the frequency of oscillation of these two modes decreases as Weber number increases, and becomes zero at the critical values. At higher Weber number the bubble motion is unstable because the real part of one eigenvalue becomes positive.

The fact that the frequency of oscillation decreases in a straining flow has an 


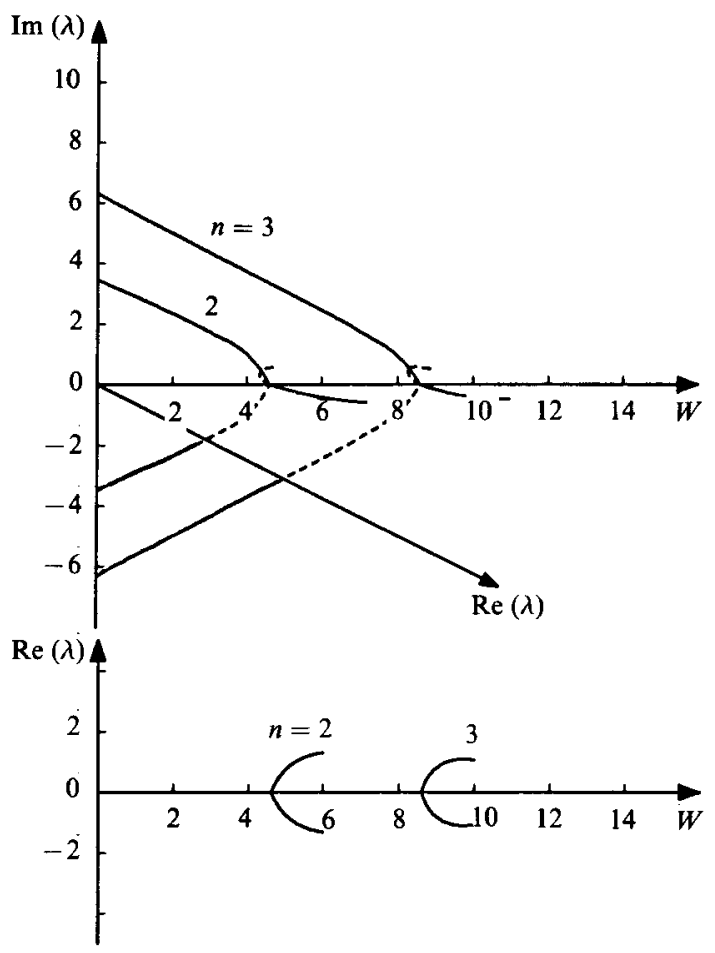

Figure 4. Eigenvalues as a function of Weber number for the $n=2$ and $n=3$ mode oscillations.

important physical significance, because it provides one example of frequency modification of a bubble in a certain type of flow. Although we do not include any steady-state shape correction in this analysis, it does provide the simplest approximation to the actual oscillatory motion of a bubble in a straining flow. In particular, though the frequency of oscillation and the critical Weber-number value will clearly deviate from the true values for a deformed bubble, the qualitative result that the frequency decreases and becomes zero at a certain critical value of $W$ will be the same. This fact is confirmed by the asymptotic analysis, with the $O(W)$ steadystate shape correction included, in $\$ 4.2$, and is also confirmed by the numerical solution of Kang \& Leal (1987). The significance of a critical Weber number, where the true frequency of oscillation becomes zero, is that this critical value will correspond exactly to a limit point of the corresponding steady-state solution. Therefore the existence of a limit point of the steady solution, which was predicted by the numerical analyses of Miksis (1981) and Ryskin \& Leal (1984), is again confirmed by a totally different approach, subject only to the assumption that the oscillatory behaviour of a spherical bubble is at least qualitatively representative of that for a real deformed bubble. The fact that the zero-frequency point is predicted here to occur at $W=4.62$, whereas the steady-state limit of convergence was found to be $W=2.76$, is presumably a consequence of the restriction to a spherical base case in the present analysis.

\subsection{Oscillation of a slightly deformed bubble for $W \ll 1$}

In $\S 4.1$, we studied the effect of straining flow on the stability and oscillatory motion of a spherical bubble. Insofar as it can be applied to a spherical bubble, our analysis 
is applicable for arbitrary Weber number. However we showed numerically (Ryskin $\&$ Leal 1984) and via the perturbation analysis of $\$ 3$ that the steady-state shape at finite Weber number is not spherical in an axisymmetric straining flow, and an immediate question arises about the effect of the non-spherical shape on the oscillatory motion. In this subsection we therefore consider the oscillatory motion of a bubble with the steady-state shape corrected to leading order (i.e. $O(W)$ ) based upon the small- $W$ expansion of $\S 3$. To be consistent with $\$ 4.1$, we should not introduce any assumption except that the steady-state shape is given by $R=1+W \zeta_{\mathrm{s}}$ $\left(\zeta_{\mathrm{s}}\right.$ is given in (3.34)) over the whole range of interesting $W$-values. However, then, the problem becomes extremely complicated because the change of steady-state shape $\left(W \zeta_{s}\right)$ requires an $O\left(W^{\frac{3}{2}}\right)$-correction of the steady-state velocity potential, whose solution is itself moderately complicated. Instead, we confine ourselves to a strict asymptotic analysis for the limit of very small Weber numbers, including all $O(W)$-terms (only) in the solution.

The analysis follows that of $\$ 4.1$ but with the steady shape function corrected to include the $O(W)$-term from $\$ 3$. Thus, the velocity potential and the shape function can be written as

$$
\begin{gathered}
\phi=\tilde{\phi}_{\mathrm{s}}+\tilde{\phi}_{\mathrm{u}}=\left(\frac{1}{2} W\right)^{\frac{1}{2}} \phi_{\mathrm{s}}+O\left(W^{\frac{3}{2}}\right)+\epsilon \phi_{\mathrm{u}}, \\
f=f_{\mathrm{s}}+f_{\mathrm{u}}=W \zeta_{\mathrm{s}}+O\left(W^{2}\right)+\epsilon \zeta_{\mathrm{u}},
\end{gathered}
$$

where

$$
\begin{aligned}
\phi_{\mathrm{s}} & =P_{2}(\eta)\left(\frac{1}{2} r^{2}+\frac{1}{3} r^{-3}\right), \\
\zeta_{\mathrm{s}} & =\delta_{2}^{(\mathrm{s}, 1)} P_{2}(\eta)+\delta_{4}^{(\mathrm{s}, 1)} P(\eta) \\
& =\frac{25}{672} P_{2}(\eta)-\frac{5}{252} P_{4}(\eta), \\
\phi_{\mathrm{u}} & =\sum_{n=0}^{\infty} \gamma_{n}(t) r^{-(n+1)} P_{n}(\eta), \\
\zeta_{\mathrm{u}} & =\sum_{n=0}^{\infty} \delta_{n}(t) P_{n}(\eta) .
\end{aligned}
$$

In (4.24) $\tilde{\phi}_{\mathrm{s}}$ and $\tilde{\phi}_{\mathrm{u}}$ denote the steady velocity potential and the disturbance velocity potential respectively, while $f_{\mathrm{s}}$ and $f_{\mathrm{u}}$ denote the steady shape function and the disturbance shape function respectively. The governing equation for the disturbance velocity potential is simply Laplace's equation. The asymptotically correct boundary conditions for an infinitesimal disturbance of $O(\epsilon)$ at small Weber number are derived as follows. First, formally perturb the kinematic condition and the normal stress condition about the steady state, $\phi=\tilde{\phi}_{\mathrm{s}}$ and $f=f_{\mathrm{s}}$ for an arbitrary fixed $W$, and retain only the $O(\epsilon)$-terms. The resulting $O(\epsilon)$-boundary conditions are partial differential equations with a single parameter $W$. To achieve the correct, leadingorder asymptotic form for the boundary conditions for $W \ll 1$, we substitute the steady-state velocity potential and the steady-state shape function $\left(\tilde{\phi}_{\mathrm{s}}=\left(\frac{1}{2} W\right)^{\frac{1}{2}} \phi_{\mathrm{s}}+\right.$ $\left.O\left(W^{\frac{3}{2}}\right), f_{\mathrm{s}}=W \zeta_{\mathrm{s}}+O\left(W^{2}\right)\right)$ into the boundary conditions, and retain terms up to $O(W)$.

The boundary conditions, at $O(\epsilon)$ and $O(W)$, obtained by the domain perturbation technique are a bit more complicated than those of the previous analysis. In particular, the kinematic condition is

$$
\frac{\partial \zeta_{\mathrm{u}}}{\partial t}=\frac{\partial \phi_{\mathrm{u}}}{\partial r}+\left(\frac{1}{2} W\right)^{\frac{1}{2}}\left(\zeta_{\mathrm{u}} \frac{\partial^{2} \phi_{\mathrm{s}}}{\partial r^{2}}-\frac{\partial \zeta_{\mathrm{u}}}{\partial \theta} \frac{\partial \phi_{\mathrm{s}}}{\partial \theta}\right)+W\left(\zeta_{\mathrm{s}} \frac{\partial^{2} \phi_{\mathrm{u}}}{\partial r^{2}}-\frac{\partial \zeta_{\mathrm{s}}}{\partial \theta} \frac{\partial \phi_{\mathrm{u}}}{\partial \theta}\right) \quad \text { at } r=1
$$


and the normal stress condition becomes

$$
\begin{aligned}
\frac{\partial \phi_{\mathrm{u}}}{\partial t} & +W \zeta_{\mathrm{s}} \frac{\partial^{2} \phi_{\mathrm{u}}}{\partial r \partial t}+\left(\frac{1}{2} W\right)^{\frac{1}{2}}\left(\nabla \phi_{\mathrm{s}} \cdot \nabla \phi_{\mathrm{u}}\right)+\frac{1}{2}\left(\frac{1}{2} W\right) \zeta_{\mathrm{u}} \frac{\partial}{\partial r}\left(\nabla \phi_{\mathrm{s}} \cdot \nabla \phi_{\mathrm{s}}\right) \\
& =\sum_{n=0}^{\infty}(n-1)(n+2) \delta_{n} P_{n}(\eta) \\
& -2 W \delta_{2}^{(\mathrm{s}, 1)} \sum_{k=0}^{\infty} \delta_{k}\left(k^{2}+k+4\right) P_{2}(\eta) P_{k}(\eta) \\
& -2 W \delta_{4}^{(\mathrm{s}, 1)} \sum_{l=0}^{\infty} \delta_{l}\left(l^{2}+l+18\right) P_{4}(\eta) P_{k}(\eta) \quad \text { at } r=1 .
\end{aligned}
$$

Also we must use the fact that the volume of the bubble is conserved and that the bubble centre remains at the origin. These conditions can be easily derived from (2.10) and (2.11):

$$
\begin{aligned}
& \int_{-1}^{1}\left(\zeta_{\mathrm{u}}+2 W \zeta_{\mathrm{s}} \zeta_{\mathrm{u}}\right) \mathrm{d} \eta=0 \\
& \int_{-1}^{1} \eta\left(\zeta_{\mathrm{u}}+3 W \zeta_{\mathrm{s}} \zeta_{\mathrm{u}}\right) \mathrm{d} \eta=0
\end{aligned}
$$

Here we note that these latter conditions were satisfied in the analysis of $\$ 4.1$ by letting $\delta_{0}=\delta_{1}=0$ in $(4.9)$.

By a similar procedure to that shown in $\$ 4.1$, we can derive the following asymptotic equations for the amplitude functions $\delta_{n}(t)$, valid up to $O(W)$ (or $\left.O\left(\xi^{2}\right)\right)$ :

$$
\begin{gathered}
\delta_{0}(t)=-\frac{16}{25} \xi^{2}\left(\frac{1}{5} \delta_{2}^{(\mathrm{s}, 1)} \delta_{2}(t)+\frac{1}{9} \delta_{4}^{(\mathrm{s}, 1)} \delta_{4}(t)\right), \\
\delta_{1}(t)=-\frac{48}{25} \xi^{2}\left[\frac{9}{35} \delta_{3}(t) \delta_{2}^{(\mathrm{s}, 1)}+\left(\frac{4}{21} \delta_{3}(t)+\frac{5}{33} \delta_{5}(t)\right) \delta_{4}^{(\mathrm{s}, 1)}\right]
\end{gathered}
$$

and

$$
\begin{array}{r}
\ddot{\delta_{n}}+\left(\left(n^{2}-1\right)(n+2)-\xi^{2}\left(G_{+0}(n)+\Delta(n)\right)\right) \delta_{n}=-\xi\left(J_{+2}(n) \dot{\delta}_{n+2}-J_{-2}(n) \dot{\delta}_{n-2}\right) \\
-\xi^{2}\left(\sum_{\substack{k=-2, k \neq 0}}^{+2} D_{2 k} \delta_{n+2 k}\right) \text { for } n \geqslant 2,
\end{array}
$$

where

$$
\begin{aligned}
\Delta(n)= & \frac{8}{25}\left(\left(n^{2}-1\right)(n+2)\left(\delta_{2}^{(\mathrm{s}, 1)} Q_{0}(n)+\delta_{4}^{(\mathrm{s}, 1)} R_{0}(n)\right)\right. \\
= & (n-1)(n+2)\left(\delta_{2}^{(\mathrm{s}, 1)} S_{0}(n)+\delta_{4}^{(\mathrm{s}, 1)} T_{0}(n)\right) \\
& \left.\quad+2 \delta_{2}^{(\mathrm{s}, 1)}(n+1)\left(n^{2}+n+4\right) Q_{0}(n)+2 \delta_{4}^{(\mathrm{s}, 1)}(n+1)\left(n^{2}+n+18\right) R_{0}(n)\right),
\end{aligned}
$$

with

$$
\begin{aligned}
& Q_{0}(n)=\frac{2 n+1}{2} \int_{-1}^{1} P_{2} P_{n} P_{n} \mathrm{~d} x, \\
& R_{0}(n)=\frac{2 n+1}{2} \int_{-1}^{1} P_{4} P_{n} P_{n} \mathrm{~d} x \\
& S_{0}(n)=\frac{2 n+1}{2} \int_{-1}^{1}\left(1-x^{2}\right) P_{2}^{\prime} P_{n}^{\prime} P_{n} \mathrm{~d} x, \\
& T_{0}(n)=\frac{2 n+1}{2} \int_{-1}^{1}\left(1-x^{2}\right) P_{4}^{\prime} P_{n}^{\prime} P_{n} \mathrm{~d} x .
\end{aligned}
$$


In (4.30) the $D_{2 k}$ are very complicated functions of $n$ of $O\left(n^{3}\right)$, which are not given here explicitly because the effect of the terms involving these coefficients will be shown below to be at most $O\left(\xi^{3}\right)$ (or $\left.O\left(W^{\frac{3}{2}}\right)\right)$.

To evaluate the eigenvalues of the system of equations (4.30), we assume that the solution can be expressed as

$$
\delta_{n}=\delta_{n 0} \mathrm{e}^{\lambda t} .
$$

Then, by substituting (4.31) into (4.30), we get the following equation for the truncated system of equations (arbitrarily large):

$$
\boldsymbol{A} \cdot \boldsymbol{x}=\mathbf{0},
$$

where the elements of $\boldsymbol{A}$ and $\boldsymbol{x}$ are given by

$$
\begin{aligned}
a_{i, i} & =\lambda^{2}+\left(\left(4 i^{2}-1\right)(2 i+2)-\xi^{2}\left(G_{0}(2 i)+4(2 i)\right)\right), \\
a_{i, i+1} & =\lambda \xi_{+2}(2 i)+D_{2}(2 i) \xi^{2} \\
a_{i, i+2} & =D_{4}(2 i) \xi^{2} \\
a_{i, i-1} & =-\lambda \xi J_{-2}(2 i)+D_{-2}(2 i) \xi^{2} \\
a_{i, i-2} & =D_{-4}(2 i) \xi^{2} \\
a_{i, j} & =0 \quad \text { if }|i-j|>2
\end{aligned}
$$

and

$$
x_{i}=\delta_{(2 i) 0} .
$$

Then the characteristic equation for the eigenvalues is given by

$$
\operatorname{det}(\boldsymbol{A})=0
$$

By definition, we can calculate the determinant as

$$
\operatorname{det}(\boldsymbol{A})=\sum_{\left(k_{1}, \ldots, k_{N}\right)} \epsilon\left(k_{1}, \ldots, k_{N}\right) a_{1 k_{1}} \ldots a_{N k_{N}}
$$

where the summation extends over all $N$ ! arrangements $\left(k_{1}, \ldots, k_{N}\right)$ of $(1, \ldots, N)$ and $\epsilon\left(k_{1}, \ldots, k_{N}\right) \equiv \operatorname{sgn} \Pi_{1 \leqslant r \leqslant s \leqslant N}\left(k_{s} \ldots k_{r}\right)$. Now, we can show, for any $N \times N$ matrix, that the number of off-diagonal elements in the term $a_{1 k_{1}} \ldots a_{N k_{N}}$ of (4.34) cannot be one. To show that, let us consider any off-diagonal element in the term $a_{1 k_{1}} \ldots a_{N k_{N}}$ (say $a_{i k_{i}}$ for $i$ th row and $k_{i}$ th column, $i \neq k_{i}$ ). Then for the $k_{i}$ th row we cannot take $a_{k_{i} k_{i}}$ (diagonal element), because any two elements in the term $a_{1 k_{1}} \ldots a_{N k_{N}}$ cannot come from the same column. Therefore we must pick one of the off-diagonal elements for the $k_{i}$ th row. This proves that the number of off-diagonal element in $a_{1 k_{1}} \ldots a_{N k_{N}}$ of (4.34) cannot be one.

Two important consequences can be drawn from this fact. First, the effect of offdiagonal elements is at most $O\left(\xi^{2}\right)$ (or $O(W)$ ), which applies to the discussion following $(4.20)$ in $\$ 4.1$. Therefore, there is no $O\left(W^{\frac{1}{2}}\right)$-term in the asymptotic expression for the oscillation frequency as a function of Weber number. Secondly, the effect of the $D_{2 k}$ in (4.30) is at most $O\left(\xi^{3}\right)$ because $D_{2 k} \xi^{2}$ must be multiplied by at least one of the other off-diagonal elements, which are at most $O(\xi)$. Therefore, for our asymptotic analysis, which is rigorous up to $O\left(\xi^{2}\right)$, we can neglect the terms in $(4.30)$ that involve the $D_{2 k}$. 
Let us return to the asymptotic solution of (4.30) for small $W$. For this purpose, we retain only terms in $(4.30)$ up to $O(W)$. The resulting equation is

$$
\begin{aligned}
& \ddot{\delta}_{n}+\left(\left(n^{2}-1\right)(n+1)-\xi^{2}\left(G_{+0}(n)+\Delta(n)\right)\right) \delta_{n} \\
& \quad=-\xi\left(J_{+2}(n) \dot{\delta}_{n+2}-J_{-2}(n) \dot{\delta}_{n-2}\right) \quad \text { for } n \geqslant 2 .
\end{aligned}
$$

Since the coefficient of $\delta_{n}$ in (4.35) is positive for $W \ll 1$, where the present analysis is intended to apply, (4.35) is of the same type as (4.15) in $\$ 4.1$, and the same procedure can be used to prove that the eigenvalues are again purely imaginary. Indeed, in this case, if we consider the truncated system involving only the $n=2$ and $n=4$ modes, we obtain the same result as before, $(4.20)$, but with the $O(W)$ correction to the oscillation frequency now evaluated:

$$
\begin{aligned}
& -\lambda_{2}^{2}=\omega_{2}^{2} \approx 12\left(1-\left(\frac{G_{0}(2)+\Delta(2)}{12}\right) \xi^{2}-\frac{\xi^{2}}{28.665}\right)=12(1-0.31 \mathrm{~W}), \\
& -\lambda_{4}^{2}=\omega_{4}^{2} \approx 90\left(1-\left(\frac{G_{0}(4)+\Delta(4)}{90}\right) \xi^{2}+\frac{\xi^{2}}{28.665}\right)=90(1+0.016 \mathrm{~W}),
\end{aligned}
$$

where

$$
\begin{array}{ll}
G_{0}(2)=0.776, & \Delta(2)=-0.00635, \\
G_{0}(4)=2.12, & \Delta(4)=0.555 .
\end{array}
$$

Although these results were obtained by considering the interaction between only the first two even modes, it can be rigorously shown that the inclusion of higher-order modes does not affect the asymptotic formula (4.36) for the $n=2$ mode up to $O\left(\xi^{2}\right)$.

To show that this is true, let us consider again the characteristic equation for the eigenvalues of the truncated system of $(4.35)$. Then by a procedure similar to that used earlier, we can show that the characteristic equation is given by a determinant of a tridiagonal matrix. If we denote the determinant of the $m \times m$ tridiagonal matrix as $|\boldsymbol{A}|_{m}$, then all we must show is that $|\boldsymbol{A}|_{N}=o\left(\xi^{2}\right)$ for any $N \geqslant 2$, provided that $|\boldsymbol{A}|_{1}=O\left(\xi^{2}\right)$ and $|\boldsymbol{A}|_{2}=o\left(\xi^{2}\right)$ (these last two conditions are satisfied by (4.36)). To do so, assume $|\boldsymbol{A}|_{k-1} \leqslant O\left(\xi^{2}\right)$ and $|\boldsymbol{A}|_{k}=o\left(\xi^{2}\right)$. Then

$$
\begin{aligned}
|\boldsymbol{A}|_{k+1} & =-a_{k, k+1} a_{k+1, k}|\boldsymbol{A}|_{k-1}+a_{k+1, k+1}|\boldsymbol{A}|_{k} \\
& =O(\xi) O(\xi) O\left(\xi^{2}\right)+O(1) o\left(\xi^{2}\right) \\
& =o\left(\xi^{2}\right)
\end{aligned}
$$

and for $k=2$, the assumption is true. This proves that (4.36) is asymptotically rigorous up to $O\left(\xi^{2}\right)$.

One interesting result from the analysis of the subsection, which is asymptotically rigorous to $O(W)$, is that the frequency of the principal mode (i.e. $n=2$ or $n=3$ ) decreases as Weber number increases, thus corroborating the ad hoc numerical result for a spherical bubble that was obtained in $\S 4.1 .3$. We shall present a comparison of all results obtained in $\$ \S 4.1$ and 4.2 later in $\S 5$. For now, let us simply note that the asymptotic formula, extrapolated to $W \sim O(1)$, suggests that the frequency $\omega_{2}$ will go to zero at $W \sim 3.2$. It is interesting that this value is closer to the numerical limit point of 2.76, obtained by Miksis (1981) and Ryskin \& Leal (1984), than the value 4.62 obtained in the preceding section via numerical analysis for a spherical bubble. 


\subsection{The influence of weak viscous effects on bubble oscillation}

From the analysis of $\$ 4.2$ we found that the frequency of oscillation decreases with increasing Weber number in an inviscid straining flow. In this section, we explore the effect of a small viscous contribution to bubble oscillation in a uniaxial straining flow. Here we again introduce the $a d$ hoc approximation that the steady-state shape is spherical for the whole Weber-number range of interest, as in \$4.1.3.

If viscous forces are very small relative to non-viscous forces, then viscous effects will be confined to a very thin boundary layer near the bubble surface. If the interface separates two liquids, or a gas and a liquid with appreciable kinematic viscosity in the gas, explicit account must be taken of the boundary layer. When the bubble has a negligible kinematic viscosity or is a void, however, viscous effects are extremely weak and a first approximation to their effect on bubble motion can be obtained from the inviscid solution. One approach, first used by Lamb (1932), is to use the inviscid solution in the whole fluid to estimate the viscous dissipation associated with bubble oscillation (Lamb 1932; Miller and Scriven 1968). However, there is a difficulty in application of this dissipation method to the present problem, because the total kinetic energy of the external flow is not defined. In the present analysis, we therefore use an alternative method which is equivalent to Lamb's dissipation method, in which we ignore the boundary layer and use the potential-flow solution right up to the boundary, with the effect of viscosity included by adding a viscous pressure correction and the viscous stress term to the normal stress balance, using the inviscid flow solution to estimate their values.

The normal stress condition, with the pressure correction and the viscous stress terms included, is given by

$$
-p_{\mathrm{v}}^{\prime}+\left(G^{\prime}\left(t^{\prime}\right)+\rho \frac{\partial \phi^{\prime}}{\partial t^{\prime}}+\frac{1}{2} \rho \nabla \phi^{\prime} \cdot \nabla \phi^{\prime}\right)+2 \mu \frac{\partial^{2} \phi^{\prime}}{\partial n^{\prime 2}}=\gamma(\nabla \cdot n) \quad \text { at } r^{\prime}=a+f^{\prime}\left(\theta, t^{\prime}\right),
$$

where $p_{\mathrm{v}}^{\prime}$ is the pressure correction due to viscosity and $\partial / \partial n^{\prime}$ denotes differentiation in the direction of the outward normal vector (cf. (2.3) for comparison). Nondimensionalization with the characteristic scales (2.5) gives

$$
-p_{\mathrm{v}}+\left(G(t)+\frac{\partial \phi}{\partial t}+\frac{1}{2} \nabla \phi \cdot \nabla \phi\right)+2 S \frac{\partial^{2} \phi}{\partial n^{2}}=(\nabla \cdot \boldsymbol{n}) \quad \text { at } r=1+f(\theta, t),
$$

where the dimensionless parameter $S$ is defined as

$$
S=\frac{\mu \phi_{\mathrm{c}} / a^{2}}{\rho \phi_{\mathrm{c}} / t_{\mathrm{c}}}=\frac{t_{\mathrm{c}}}{a^{2} / \nu}=\frac{t_{\mathrm{c}, \mathrm{sur}}}{t_{\mathrm{c}, \mathrm{vis}}}
$$

It is evident that $S$ can be interpreted as the ratio of a surface-tension-based timescale and the viscosity diffusion time, $a^{2} / \nu . S$ also can be expressed in terms of the Weber and Reynolds numbers as

$$
S=\frac{(2 W)^{\frac{1}{2}}}{R e}
$$

where Reynolds number $R e$ is defined as $R e=2 \rho E a^{2} / \mu$. In the present analysis we assume that $S \ll 1$.

As stated earlier, we consider the influence of weak viscous effects $(S \ll 1)$ on the oscillation of a bubble which is assumed to be spherical at steady state for the whole 
range of Weber numbers, $W=O(1)$. Hence, a small disturbance to the spherical shape is introduced as before,

$$
\begin{gathered}
\phi=\left(\frac{1}{2} W\right)^{\frac{1}{2}} \phi_{\mathrm{s}}+\epsilon \phi_{\mathrm{u}} \\
=\left(\frac{1}{2} W\right)^{\frac{1}{2}} P_{2}(\eta)\left(\frac{1}{2} r^{2}+\frac{1}{3} r^{-3}\right)+\epsilon \sum_{n=0}^{\infty} \gamma_{n}(t) r^{-(n+1)} P_{n}(\eta), \\
R=1+\epsilon \zeta_{\mathrm{u}}=1+\epsilon \sum_{n=2}^{\infty} \delta_{n}(t) P_{n}(\eta),
\end{gathered}
$$

but this time including the viscous terms in (4.38). The governing equation and the boundary conditions are the same as those in $\$ 4.1$ except for the normal stress condition. Following the approach of $\$ 4.1$, it would seem that a convenient form of the normal stress condition for the unsteady disturbance variables could be obtained by application of the domain perturbation technique to transform (4.38) applied at the actual, time-dependent bubble surface to an equivalent condition applied at $r=$ 1. This procedure can, in fact, be applied to all terms in (4.38) except for the viscous pressure correction, where it does not work. The problem with $p_{\mathrm{v}}(r, \theta)$ is that it is confined to a very thin boundary layer so that the usual approximation

$$
p_{\mathrm{v}}\left(1+\epsilon \zeta_{\mathrm{u}}, \theta\right)=p_{\mathrm{v}}(1, \theta)+\epsilon \zeta_{\mathrm{u}} \frac{\partial p_{\mathrm{v}}}{\partial r}(1, \theta)+\ldots
$$

breaks down $\left(\partial p_{\mathrm{v}} / \partial r\right.$ is large). In spite of this diffieulty with $p_{\mathrm{v}}$, however, it is still useful to apply the domain perturbation to all other terms in (4.38). If we do this, subtract the steady normal stress condition for the steady-state bubble shape $\dagger$ and then retain only terms of $O(\epsilon)$, we obtain

$$
\begin{aligned}
-\tilde{p}_{\mathrm{v}}+ & {\left[\frac{\partial \phi_{\mathrm{u}}}{\partial t}+\left(\frac{1}{2} W\right)^{\frac{1}{2}}\left(\nabla \phi_{\mathrm{s}} \cdot \boldsymbol{\nabla} \phi_{\mathrm{u}}\right)+\frac{1}{2}\left(\frac{1}{2} W\right) \zeta_{\mathrm{u}} \frac{\partial}{\partial r}\left(\nabla \phi_{\mathrm{s}} \cdot \boldsymbol{\nabla} \phi_{\mathrm{s}}\right)\right.} \\
& \left.+2 S\left(\frac{\partial^{2} \phi_{\mathrm{u}}}{\partial r^{2}}+\zeta_{\mathrm{u}}\left(\frac{1}{2} W\right)^{\frac{1}{2}} \frac{\partial}{\partial r}\left(\frac{\partial^{2} \phi_{\mathrm{s}}}{\partial r^{2}}\right)+2\left(\frac{1}{2} W\right)^{\frac{1}{2}}\left(\frac{\partial \zeta_{\mathrm{u}}}{\partial \theta}\right)\left(\frac{\partial \phi_{\mathrm{s}}}{\partial \theta}\right)\right)\right]_{r=1} \\
= & \sum_{n=2}^{\infty}(n-1)(n+2) \delta_{n} P_{n}(\eta)
\end{aligned}
$$

Here, $\tilde{p}_{\mathrm{v}}$ is the disturbance of the pressure correction due to unsteady deformation which can be expressed in the form

$$
\tilde{p}_{\mathrm{v}}=\frac{1}{\epsilon}\left(p_{\mathrm{v}}\left(1+\epsilon \zeta_{\mathrm{u}}, \theta\right)-p_{\mathrm{v}}^{\mathrm{s}}(1, \theta)\right)
$$

where $p_{\mathrm{v}}\left(1+\epsilon \zeta_{\mathrm{u}}, \theta\right)$ denotes the pressure correction due to viscosity at the perturbed, unsteady bubble surface, and $p_{v}^{\mathrm{s}}(1, \theta)$ denotes the steady-state viscous pressure correction at the spherical bubble surface.

The viscous pressure correction $p_{v}\left(1+\epsilon \zeta_{u}, \theta\right)$ in (4.41) should be obtained, in principle, by solving the Navier-Stokes equation with boundary conditions applied at the actual deformed bubble surface $\left(R=1+\epsilon \zeta_{u}\right)$. In practice, however, this is extremely difficult for the high-Reynolds-number problem, because the usual

$\dagger$ In spite of the fact that the steady-state normal stress balance is not exactly satisfied for the assumed spherical steady-state base shape, we subtract the full steady-state condition to remove terms that would otherwise act to superpose a time-dependent evolution toward the correct steady shape onto the time-dependent oscillations of shape that concern us here. 
domain perturbation technique is not again applicable to the tangential stress condition (the tangential stress has a singular-like behaviour near the boundary at high Reynolds numbers that is very similar to $p_{\mathrm{v}}$ ). As a consequence, in the present work, we adopt the ad hoc approximation of applying the tangential stress condition at the undeformed spherical surface $(R=1)$ rather than at the actual deformed bubble surface $\left(R=1+\epsilon \zeta_{u}\right)$. This approximation results in an error in the viscous pressure correction of $O\left(\epsilon p_{\mathrm{v}}^{*}(1, \theta)\right)$ (where $p_{\mathrm{v}}^{*}(1, \theta)$ denotes the pressure correction obtained with the approximation), i.e.

$$
p_{\mathrm{v}}\left(1+\epsilon \zeta_{\mathrm{u}}, \theta\right)=p_{\mathrm{v}}^{*}(1, \theta)+\epsilon \zeta_{\mathrm{u}} O\left(p_{\mathrm{v}}^{*}(1, \theta)\right) .
$$

The effect of the error terms will be discussed later.

A general formula relating the viscous pressure correction to the vorticity distribution is derived in the Appendix for a spherical bubble in an arbitrary axisymmetric flow. This formula can be applied in the present problem to evaluate $p_{\mathrm{v}}^{*}(1, \theta)$ and $p_{\mathrm{v}}^{\mathrm{s}}(1, \theta)$, and thus via the approximation $(4.42)$ we can approximate $\tilde{p}_{\mathrm{v}}$ in (4.40). Since we ignore the boundary layer, the pressure corrections at $r=1$, obtained from the formula (A 10) derived in the Appendix, can be expressed in the forms

$$
\begin{aligned}
& p_{\mathrm{v}}^{*}(1, \theta) \approx \sum_{n=0}^{\infty} n S T_{n}(1) P_{n}(\cos \theta), \\
& p_{\mathrm{v}}^{\mathrm{s}}(1, \theta) \approx \sum_{n=0}^{\infty} n S T_{n}^{\mathrm{s}}(1) P_{n}(\cos \theta) .
\end{aligned}
$$

The coefficients $T_{n}(1)$ in (4.43) are obtained from the vorticity value at $r=1$,

$$
\omega=-\sum_{n=0}^{\infty} \frac{T_{n}}{r} \frac{\mathrm{d} P_{n}}{\mathrm{~d} \theta}=\frac{\partial u_{\theta}}{\partial r}+\frac{u_{\theta}}{r}-\frac{1}{r} \frac{\partial u_{r}}{\partial \theta}
$$

and the tangential stress condition which must be satisfied at $r=1$ :

$$
\frac{\partial u_{\theta}}{\partial r}-\frac{u_{\theta}}{r}+\frac{1}{r} \frac{\partial u_{r}}{\partial \theta}=0
$$

By eliminating $\partial u_{\theta} / \partial r$, we get

$$
-\sum_{n=0}^{\infty} \frac{T_{n}}{r} \frac{\mathrm{d} P_{n}}{\mathrm{~d} \theta}=2\left(\frac{u_{\theta}}{r}-\frac{1}{r} \frac{\partial u_{r}}{\partial \theta}\right) \approx 2\left(\frac{u_{\mathrm{p} \theta}}{r}-\frac{1}{r} \frac{\partial u_{\mathrm{p} r}}{\partial \theta}\right) .
$$

By substituting the potential-flow field in $(4.39 a)$ into $(4.46)$, and by comparing the coefficients of $\mathrm{d} P_{n} / \mathrm{d} \theta$, we obtain,

$$
\begin{aligned}
T_{2}(1)=-\frac{5}{3}\left(\frac{1}{2} W\right)^{\frac{1}{2}}-2 \epsilon(n+2) \gamma_{n} & \text { for } n=2 \\
T_{n}(1)=-2 \epsilon(n+2) \gamma_{n} & \text { for } n \neq 2
\end{aligned}
$$

For the steady-state problem, we have

$$
\begin{aligned}
T_{2}^{\mathrm{s}}(1)=-\frac{5}{3}\left(\frac{1}{2} W\right)^{\frac{1}{2}} & \text { for } n=2 \\
T_{n}^{\mathrm{s}}(1)=0 & \text { for } n \neq 2 .
\end{aligned}
$$

Thus, using (4.42), (4.43), and (4.47), we can estimate the disturbance of the viscous pressure $\tilde{p}_{\mathrm{v}}$ in $(4.41)$ as

$$
\tilde{p}_{\mathrm{v}}=-(2 S) \sum_{n=2}^{\infty} n(n+2) \gamma_{n} P_{n}+\zeta_{\mathrm{u}} O\left(S W^{\frac{1}{2}}\right)
$$


Then, the normal stress condition (4.40) can be written as

$$
\begin{aligned}
& \sum_{n=2}^{\infty}\left[\dot{\gamma}_{n}+(2 S)(2 n+1)(n+2) \gamma_{n}\right] P_{n}(\eta)+\left(\frac{1}{2} W\right)^{\frac{1}{2}}\left(\nabla \phi_{\mathrm{s}} \cdot \boldsymbol{\nabla} \phi_{\mathrm{u}}\right) \\
& \quad=\sum_{n=2}^{\infty}\left[(n-1)(n+2)-O\left(S W^{\frac{1}{2}}\right)-\frac{1}{2}\left(\frac{1}{2} W\right) \frac{\partial}{\partial r}\left(\boldsymbol{\nabla} \phi_{\mathrm{s}} \cdot \boldsymbol{\nabla} \phi_{\mathrm{s}}\right)\right] \delta_{n} P_{n}(\eta) \quad \text { at } r=1 .
\end{aligned}
$$

Although we do not know the details of the $O\left(S W^{\frac{1}{2}}\right)$-term, it can be safely neglected if $S \ll 1$ and $W^{\frac{1}{2}} \ll 1$ because $S W^{\frac{1}{2}} \ll S$. If $W^{\frac{1}{2}}=O(1)$ and $S \ll 1$, on the other hand, the $O\left(S W^{\frac{1}{2}}\right)$-term is apparently comparable with the $O(S)$-term. However, the roles of the two terms are totally different. In particular, the $O\left(S W^{\frac{1}{2}}\right)$-term yields a negligible correction to the oscillation frequency, while the $O(S)$-term contributes to viscous damping at the leading order. Therefore, in deriving an equation for the amplitude of shape coefficients, $\delta_{n}(t)$, it is not unreasonable to neglect the $O\left(S W^{\frac{1}{2}}\right)$-term up to $W \sim O(1)$, as long as $S \ll 1$.

Now, we obtain a modified version of (4.14) by a similar procedure to that shown in $\$ 4.1$, but this time including a viscous damping term,

$$
\begin{aligned}
\ddot{\delta}_{n}+ & (2 S)(2 n+1)(n+2) \dot{\delta}_{n}+\left(\left(n^{2}-1\right)(n+2)-\xi^{2} G_{0}(n)\right) \delta_{n} \\
& =-\xi\left(J_{+2}(n) \dot{\delta}_{n+2}-J_{-2}(n) \dot{\delta}_{n-2}\right) \\
& -\xi^{2}\left(G_{+4}(n) \delta_{n+4}-G_{+2}(n) \delta_{n+2}-G_{-2}(n) \delta_{n-2}+G_{-4}(n) \delta_{n-4}\right)
\end{aligned}
$$

where $\xi=\frac{5}{2}\left(\frac{1}{2} W\right)^{\frac{1}{2}}$, and the other coefficients are given in (4.14).

As in $\S 4.1$, it is advantageous to begin by studying the asymptotic behaviour of (4.50) for several limiting cases. First, we study the asymptotic form for the limit $n \rightarrow \infty$, and then, the asymptotic form for asymptotically very small Weber numbers. Following this, we study the behaviour of $(4.50)$ for wide range of Weber numbers but small $S$, by evaluating the eigenvalues numerically.

\subsubsection{Asymptotic form for the limit $n \rightarrow \infty$}

For the limit $n \rightarrow \infty$, if we rescale time as $\tilde{t}=\omega t=n^{\frac{3}{2}} t,(4.50)$ is reduced to the form

$$
\frac{\mathrm{d}^{2} \delta_{n}}{\mathrm{~d} \tilde{t}^{2}}+(4 S) n^{\frac{1}{2}} \frac{\mathrm{d} \delta_{n}}{\mathrm{~d} \tilde{t}}+\delta_{n}=0 \quad \text { as } n \rightarrow \infty
$$

The physical significance of this result is that the high-frequency modes are damped out faster than the low-frequency modes. It may also be noted that only aperiodic damping is possible for all high-frequency modes with $n>n_{\mathrm{c}}=1 / 4 \mathcal{S}^{2}$.

\subsection{2. $\xi \ll S \ll 1$ case}

In this limit, the effect of the straining flow can be neglected $(\xi=0)$. The result (4.50) for $\xi=0$ is identical with that obtained many years ago by Lamb (1932) using the dissipation argument that was cited earlier. For this zero-mean-flow limit, it is well known that only aperiodic damping is possible for $S \geqslant\left\{\left(n^{2}-1\right) /(n+2)\right.$ / $\left.(2 n+1)^{2}\right\}^{\frac{1}{2}}($ e.g. $S \geqslant 0.173$ for $n=2)$.

\subsubsection{Numerical evaluation of eigenvalues for larger Weber numbers but small $S$}

Finally, the eigenvalue for the $n=2$ mode is numerically evaluated for a wide range of Weber numbers. The real and the imaginary parts are shown in figure 5 for several values of $S$. For a very small $S(S<0.05)$, the solution behaviour is more or 

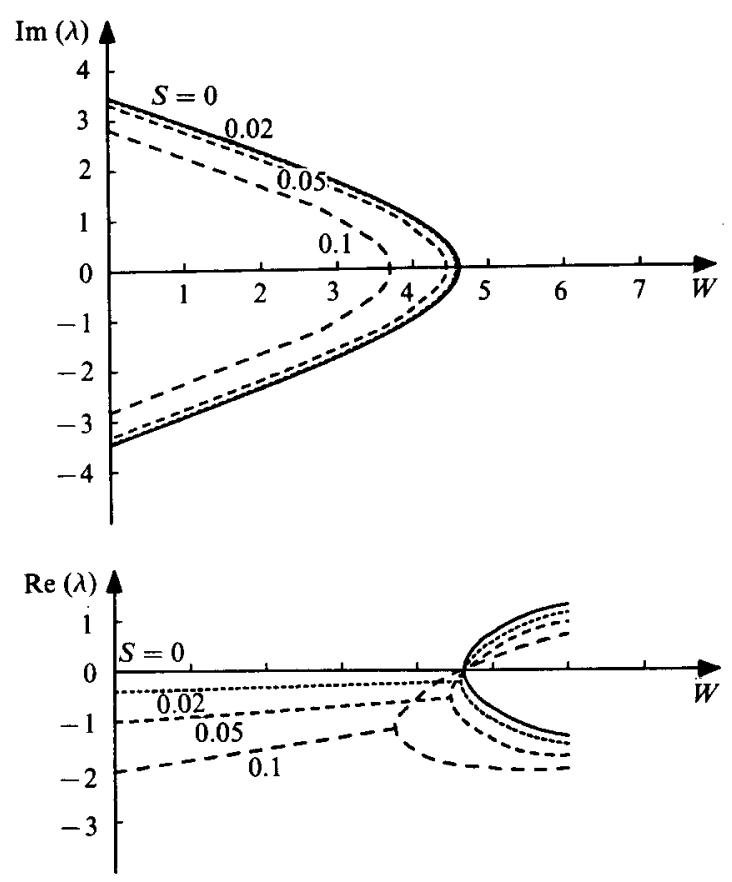

Figure 5. The effect of small viscosity on the eigenvalues for the $n=2$ mode oscillation.

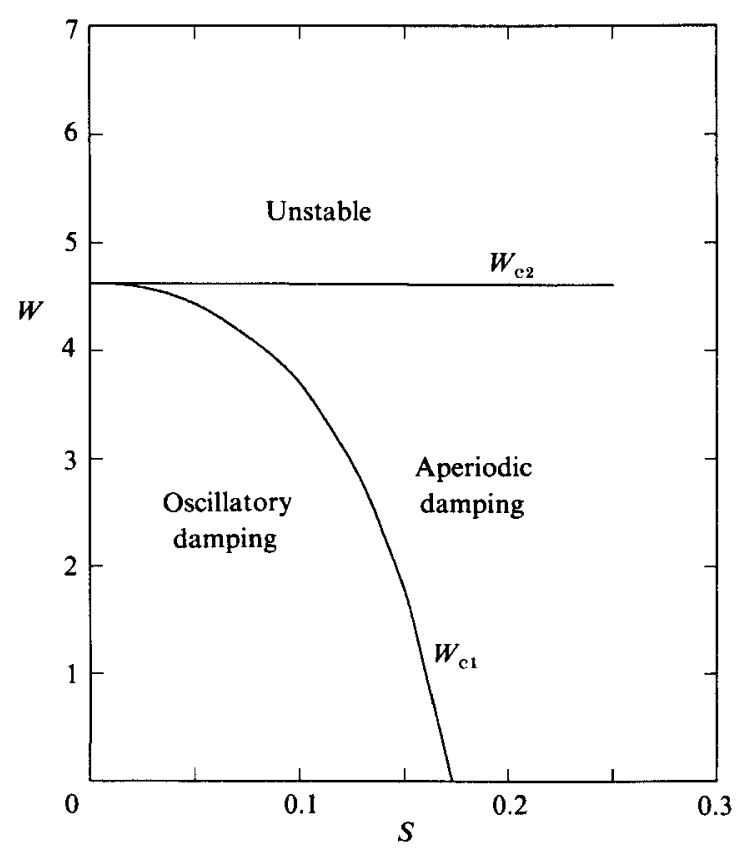

Figure 6. Three different types of bubble motion in a slightly viscous straining flow. 
less the same as in the zero-viscosity case except that the oscillation is weakly damped. In particular, the frequencies of oscillation are almost the same as in the inviscid case. The numerical results also show that the eigenvalues can be either complex numbers with negative real parts, or pure real numbers. For a given $S$ below 0.173 , two eigenvalues for the principal mode $(n=2)$ are complex numbers with negative real parts in the range of $0 \leqslant W \leqslant W_{\mathrm{c1}}$, two are pure negative real numbers for $W_{\mathrm{c} 1} \leqslant W \leqslant W_{\mathrm{c} 2}$, and one is a negative real number but the other is a positive real number for $W>W_{\text {c2 }}$. Therefore, for $S<0.173$, a bubble can undergo oscillatory damping, or aperiodic damping, or an unstable deformation, depending on the value of Weber number.

The two critical values of $W_{\mathrm{c} 1}$ and $W_{\mathrm{c} 2}$ for the principal mode are shown as a function of $S$ in figure 6 . From this figure we can see that the critical value for $W_{c 1}$ decreases as $S$ increases, as expected, and that an oscillatory damping does not exist for $S>0.173$. The critical value $W_{\mathrm{c} 2}$ for instability, on the other hand, is almost constant, independent of $S$. Here we must note that we have used the ad hoc assumption of a spherical base state and neglected $O\left(S W^{\frac{1}{2}}\right)$-terms in the analysis. If we included the steady-state shape effect and /or the $O\left(S W^{\frac{1}{2}}\right)$-terms, then possibly different results for $W_{\mathrm{c} 2}$ would be obtained. Kang \& Leal's (1987) full time-dependent numerical analysis showed that $W_{\mathrm{c} 2}$ is a decreasing function of $S$ ( $W_{\mathrm{c} 2} \sim 2.8$ for $S=$ $0, W_{\mathrm{c} 2} \sim 2.2$ for $S=0.021$, and $W_{\mathrm{c} 2} \sim 1.0$ for $S=0.14$ ).

\section{Discussion of Results}

\subsection{Accuracy of the steady-state solution}

In figure 7 , we compare the shape function $f(\equiv R-1)$ at the stagnation points (at $\theta=0$ and $\left.\theta=\frac{1}{2} \pi\right)$, as estimated from the first-order and the second-order $W$ perturbation solution, with the numerical solutions of Ryskin \& Leal (1984). The upper limit on $W$ corresponds to the point beyond which a numerical solution of the steady-state problem could not be achieved. The second-order $W$-perturbation solution shows very good agreement with the numerical solution for $W \leqslant 1.5$, but the discrepancy for higher Weber numbers clearly shows the need for higher-order terms in order to get accurate results for higher Weber numbers (though it is possible that no finite number of terms would give good results for $W \approx W_{\mathrm{c}}$; see $\S 3$ ). The first-order solution agrees very well with the numerical solution up to $W=0.5$, and shows reasonable agreement up to $W=1$. Since we used the first-order steady-state solution to derive the asymptotic formula for frequency change at small Weber number, the accuracy of the first-order steady-state solution provides valuable insight into the range of Weber numbers where the asymptotic formula can be expected to give reasonable quantitative results. On this basis, the asymptotic formula for the oscillation frequency should be accurate at least up to $W=0.5$, and reasonably good for even higher Weber numbers.

\subsection{Small-amplitude oscillation about the steady-state shape}

In figure 8 , we plot the square of the frequency for the $n=2$ mode $\left(\omega_{2}^{2}\right)$ as predicted by the perturbation analysis, together with the numerical results of Kang \& Leal (1987). As shown in the previous section, the small-amplitude analysis is performed in two ways. In the first approach, we assume that the steady-state shape is spherical for the complete Weber-number range of interest. The curved broken line in figure 8 


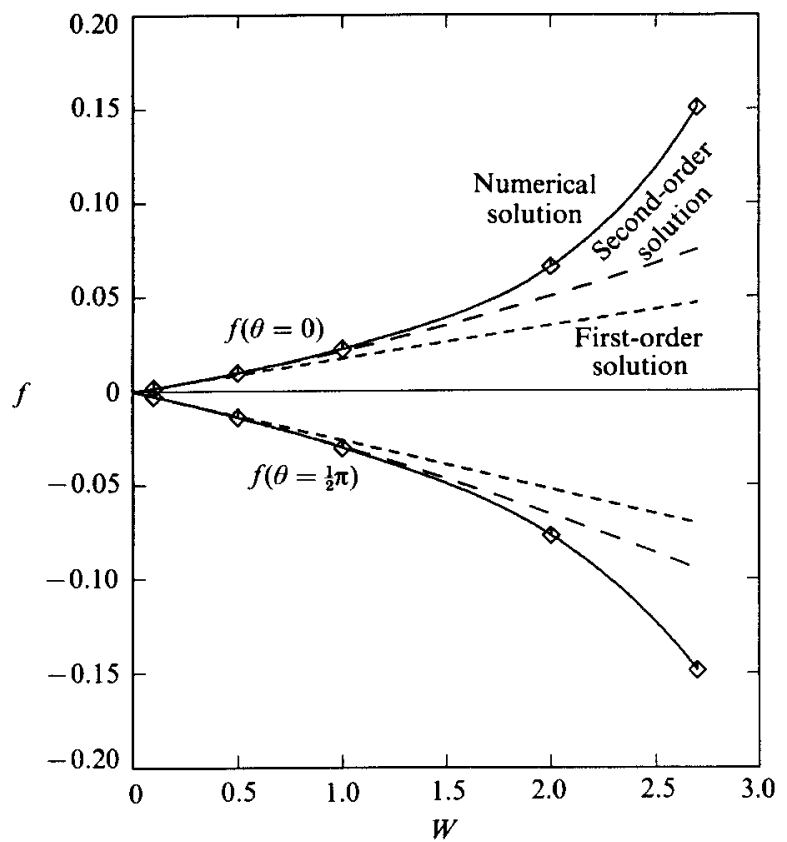

Figure 7. Comparison of the present results for steady shape with the numerical-analysis results of Ryskin \& Leal (1984):-- - - first-order perturbation solution; - $-\longrightarrow$ - the second-order perturbation solution; $\$$, numerical solution.

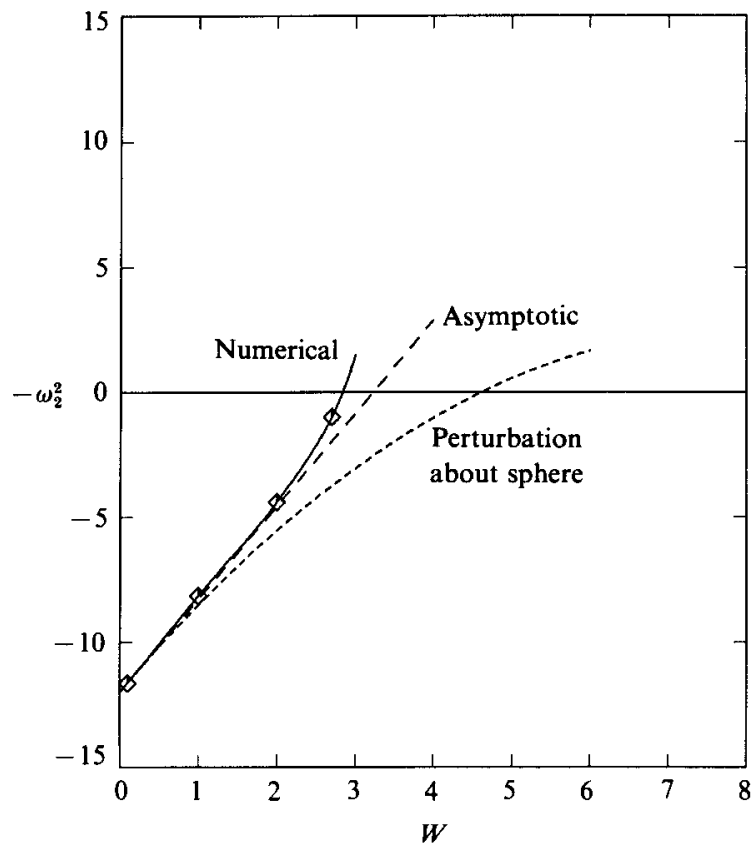

Figure 8. Comparison of the perturbation solution with the numerical-analysis results of Kang \& Leal for the square of eigenvalues of the $n=2$ mode oscillation: $\$$, numerical solution; --- , perturbation solution for the oscillation about the spherical base shape; ---- , asymptotic solution for the oscillation about the steady-state shape, $\omega^{2}=\omega_{0}^{2}(1-0.31 \mathrm{~W})$. 
represents the numerically evaluated frequencies in this analysis. Since the true steady-state shape deviates from spherical as Weber number increases, a quantitative discrepancy between this spherical solution and the numerical result is not surprising for the higher Weber-number region, as is indeed shown in figure 8 . The critical Weber number at which the frequency becomes zero is found to be 4.62 for this perturbation about a sphere. In contrast, the numerical solution shows that the critical value should be between 2.7 and 2.8 , if bubble deformation were taken into account. The relatively large discrepancy between these values is clearly due to the neglect of deformation in the steady-state shape. Note, however, that the qualitative effect of the flow on the oscillation frequency is more or less the same as that found numerically.

The second result shown in figure 8 is the rigorous asymptotic analysis, where the steady-state shape deformation is included up to $O(W)$. The simple asymptotic formula obtained for the frequency change of the $n=2$ mode is $\omega_{2}^{2}=12(1-0.31 \mathrm{~W})$, which is shown as the dotted straight line in figure 8 . This asymptotic formula shows excellent agreement with the numerical solution up to $W \approx 2$, and the predicted critical value 3.2 is much closer to the numerically obtained result.

It is worthwhile to note that the existence of a zero-frequency Weber number is equivalent to the existence of a limit point for the steady-state solution. In fact, the critical value for zero frequency predicted numerically by Kang \& Leal (1987) is precisely the same as the limit point of the steady-state solution found numerically by Miksis (1981) and Ryskin \& Leal (1984). The reason why the zero-frequency point is equivalent to the limit point of the steady-state solution is very clear. Since the small-amplitude oscillation corresponds to the unsteady behaviour of a small disturbance to the steady-state solution, the Jacobian matrix for the small perturbation clearly must become singular at the zero-frequency point (or zeroeigenvalue point). In addition, we can show that the limit point is a turning point. Therefore the steady-state solution of the branch emanating from the spherical shape for $W=0$ does not exist for $W>W_{\mathrm{c}}$ in the neighbourhood of the critical point (see Miksis 1981). From this point of view, the zero-frequency point (or the transition point from a purely imaginary eigenvalue to a purely real eigenvalue) obtained by the perturbation analysis is clearly an asymptotically predicted limit point for the existence of the steady-state solution, rather than a simple onset point for instability of the steady state.

\subsection{The influence of weak viscosity effects}

The analysis of weak viscous effects shows that viscosity has a stabilizing effect in damping the amplitude of oscillation. However, for a given fixed viscosity (or $S$ ), the unsteady bubble motion can be categorized as one of damped oscillation, aperiodic damping, and unstable indefinite deformation depending on the value of the Weber number. Thus, there are two kinds of critical Weber number when viscous effects are included in the analysis. They are the zero-frequency point where the bubble motion changes from oscillatory damping to aperiodic damping, and the zero-eigenvalue point where the steady-state bubble shape reaches a limit point.

\section{Conclusions}

The analyses reported in this paper have led to the following general conclusions:

(i) The steady-state analysis based on the small parameter $\epsilon=\left\langle R, P_{2}\right\rangle$ enables us to obtain an estimate of the critical Weber number beyond which no steady-state 
solution is found (e.g. the critical value 1.73 is estimated from the third-order solution).

(ii) The first-order steady solution in $W$ shows that the maximum radius occurs at $\theta=\frac{1}{6} \pi$, which clearly indicates the barrel-like steady-state shape for higher Weber number which was first obtained by the numerical analysis of Miksis (1981) and Ryskin \& Leal (1984).

(iii) The small-amplitude oscillation analysis about the spherical steady-state shape shows that a bubble still oscillates in an inviscid straining flow, but that the frequency decreases as Weber number increases. For the spherical bubble, the frequency goes to zero at $W=W_{\mathrm{c}}=4.62$. Thus, a spherical bubble in an inviscid straining flow would be unstable for Weber numbers larger than 4.62 .

(iv) The result of the asymptotic analysis for small-amplitude oscillation, including the correct steady-state shape up to $O(W)$, also shows that a bubble undergoes oscillatory motion with a reduced frequency due to the straining flow ; for example, for the $n=2$ mode, $\omega^{2}=\omega_{0}^{2}(1-0.31 \mathrm{~W})$, where $\omega_{0}$ is the oscillation frequency of the bubble in a quiescent fluid (for the same mode).

(v) When we consider weak viscous effects, we find that small-amplitude bubble motions in a uniaxial straining flow can be categorized into three types depending on the value of the Weber number: a damped oscillation for Weber numbers below the zero-frequency point (the eigenvalue becomes pure real at this point); an aperiodic damping between the zero-frequency point and the zero-eigenvalue point (at least one eigenvalue becomes zero at this point); and an unstable indefinite deformation above the zero-eigenvalue point.

This work was supported by a grant from the Fluid Mechanics Program of the National Science Foundation. The authors wish to thank Professor R. A. Brown for his insightful comments on an earlier version of this paper.

\section{Appendix: Derivation of a general formula for the viscous pressure correction for a spherical bubble in an arbitrary axisymmetric flow}

Let us begin by considering a weak viscous flow superposed on a given potential flow field.

$$
\boldsymbol{u}=\boldsymbol{u}_{\mathrm{p}}+\boldsymbol{u}_{\mathrm{v}} .
$$

Then, the Navier-Stokes equation for the viscous correction for small $S$ in dimensionless form is given by

$$
\frac{\partial \boldsymbol{u}_{\mathrm{v}}}{\partial t}+\left(\boldsymbol{u}_{\mathrm{p}} \cdot \boldsymbol{\nabla}\right) \boldsymbol{u}_{\mathrm{v}}+\left(\boldsymbol{u}_{\mathrm{v}} \cdot \boldsymbol{\nabla}\right) \boldsymbol{u}_{\mathrm{p}}=-\boldsymbol{\nabla} p_{\mathrm{v}}+S \nabla^{2} \boldsymbol{u}_{\mathrm{v}}+o\left(S^{\frac{1}{2}}\right)
$$

with the boundary conditions $\boldsymbol{u}_{\mathrm{v}} \rightarrow 0$ as $r \rightarrow \infty$, and $u_{\mathrm{v} r}=0$ at $r=1$. By taking the curl of (A 1$)$, we get the vorticity equation $\left(\omega=\nabla \times u_{\mathrm{v}}\right)$

$$
\frac{\partial \omega}{\partial t}+\nabla \times\left(\omega \times u_{\mathrm{p}}\right)=-S \nabla \times(\nabla \times \omega) .
$$

Since the vorticity field is solenoidal $(\nabla \cdot \omega=0)$, we can express the vorticity for this axisymmetric problem in terms of toroidal $(T)$ fields (Chandrasekhar 1961; Prosperetti 1977)

$$
\boldsymbol{\omega}=\boldsymbol{T}=\boldsymbol{\nabla} \times\left(\sum_{n=0}^{\infty} T_{n}(r, t) P_{n}(\cos \theta) \boldsymbol{e}_{r}\right)
$$


Also the viscous correction to the velocity field of the axisymmetric problem can be expressed, in general, as

$$
\boldsymbol{u}_{\mathrm{v}}=\left(\sum_{n=0}^{\infty} T_{n} P_{n}\right) \boldsymbol{e}_{r}-\nabla \Phi=\left(\sum_{n=0}^{\infty} T_{n} P_{n}\right) \boldsymbol{e}_{r}-\nabla\left(\sum_{n=0}^{\infty} \Phi_{n}\right)
$$

where $\Phi$ is an unknown potential function which must be determined to satisfy the incompressibility condition and the boundary conditions. From the incompressibility condition, we have

$$
\nabla^{2} \Phi_{n}=\nabla \cdot\left(T_{n} P_{n} e_{r}\right)
$$

The solution of (A 5) satisfying the kinematic condition $\left(u_{v r}(1, \theta)=0\right)$ was obtained by Prosperetti (1977):

$$
\begin{aligned}
\Phi_{n}=P_{n}\left[\left(\alpha_{n}(t)+\frac{n+1}{2 n+1} \int_{1}^{r} s^{-n} T_{n}(s, t) \mathrm{d} s\right) r^{n}\right. & \\
& \left.+\left(\frac{n}{n+1} \alpha_{n}(t)+\frac{n}{2 n+1} \int_{1}^{r} s^{n+1} T_{n}(s, t) \mathrm{d} s\right) r^{-(n+1)}\right] .
\end{aligned}
$$

The unknown coefficient $\alpha_{n}(t)$ in (A 6 ) is determined by the regularity condition at infinity:

$$
\alpha_{n}(t)=-\frac{n+1}{2 n+1} \int_{1}^{\infty} s^{-n} T_{n}(s, t) \mathrm{d} s
$$

A differential equation for $T_{n}(r, t)$ is obtained by substituting (A 3) into (A 2),

$$
\begin{aligned}
\sum_{n=0}^{\infty}\left[\frac{\partial T_{n}}{\partial t} P_{n}+\frac{\partial}{\partial r}\left(u_{\mathrm{p} r} T_{n}\right) P_{n}-\frac{\partial}{\partial r}\left(T_{n} \int_{0}^{\theta} P_{n}\right.\right. & \left.\frac{\partial u_{\mathrm{p} r}}{\partial \theta} \mathrm{d} \theta\right)+\frac{T_{n} u_{\mathrm{p} \theta}}{r} \frac{\mathrm{d} P_{n}}{\mathrm{~d} \theta} \\
& \left.+S\left(\frac{n(n+\mathbf{1})}{r^{2}} T_{n}-\frac{\partial^{2} T_{n}}{\partial r^{2}}\right) P_{n}\right]=G(r, t),
\end{aligned}
$$

where $G(r, t)$ denotes the terms that are independent of $\theta$. Finally, an equation for the viscous pressure correction $p_{\mathrm{v}}$ is obtained from (A 1), (A 4), and (A 6),

$$
\sum_{n=0}^{\infty}\left[\frac{\partial T_{n}}{\partial t} P_{n}+\frac{T_{n} u_{\mathrm{p} \theta}}{r} \frac{\mathrm{d} P_{n}}{\mathrm{~d} \theta}+S \frac{n(n+1)}{r^{2}} T_{n} P_{n}\right]+\frac{\partial}{\partial r}\left(-\dot{\Phi}+u_{\mathrm{v}} \cdot u_{\mathrm{p}}+p_{\mathrm{v}}\right)=0
$$

Upon integrating (A 9) with $p_{\mathrm{v}}(\infty, \theta)=0$ and eliminating the time-derivative terms, we get

$$
\begin{aligned}
p_{\mathrm{v}}(1, \theta)= & \sum_{n=0}^{\infty}\left[n \int_{1}^{\infty} r^{-n-1} \sum_{k=-\infty}^{\infty} f_{n, k}(r) T_{n+k} \mathrm{~d} r\right. \\
& \left.+\int_{1}^{\infty} \sum_{l=-\infty}^{\infty}\left(\left(r^{-n} g_{n, l}(r)-r^{-n-l} g_{n, l}(1)\right) T_{n+l}\right) \mathrm{d} r+n S T_{n}(1)\right] P_{n}(\cos \theta),
\end{aligned}
$$

where

$$
\begin{gathered}
\sum_{n=0}^{\infty}\left(\sum_{k=-\infty}^{\infty} f_{n, k} T_{n+k}\right) P_{n}=\sum_{n=0}^{\infty}\left(\left(u_{\mathrm{p} r} P_{n}-\int_{0}^{\theta} P_{n} \frac{\partial u_{\mathrm{p} r}}{\partial \theta} \mathrm{d} \theta\right) T_{n}\right), \\
\sum_{n=0}^{\infty}\left(\sum_{l=-\infty}^{\infty} g_{n, l} T_{n+l}\right) P_{n}=\sum_{n=0}^{\infty}\left(\frac{T_{n}}{r} u_{\mathrm{p} \theta} \frac{\mathrm{d} P_{n}}{\mathrm{~d} \theta}\right) .
\end{gathered}
$$

The general formula (A 10) can be used to estimate the viscous pressure correction for a spherical bubble in an arbitrary axisymmetric flow field. 


\section{REFERENCES}

Annamalai, P., Trinh, E. \& Wang, T. G. 1985 Experimental study of the oscillations of the rotating drop. J. Fluid Mech. 158, 317-327.

Busse, F. H. 1984 Oscillations of a rotating liquid drop. J. Fluid Mech. 142, 1-8.

Chandrasekhar, S. 1961 Hydrodynamic and Hydromagnetic Stability. Dover.

Iooss, G. \& Joseph, D. D. 1980 Elementary Stability and Bifurcation Theory. Springer.

Kang, I. S. \& LeaL, L. G. 1987 Numerical solution of unsteady free-boundary problems at finite Reynolds number - I. Finite-difference scheme and its application to the deformation of a bubble in a uniaxial straining flow. Phys. Fluids 30, 1929-1940.

LAMB, H. 1932 Hydrodynamics. 6th edn. Dover.

Marston, P. L. 1980 Shape oscillation and static deformation of drops and bubbles driven by modulated radiation stress: theory. J. Acoust. Soc. Am. 67, 15-26.

Miksis, M. 1981 A bubble in an axially symmetric shear flow. Phys. Fluids 24, 1229-1231.

Miller, C. A. \& Scriven, L. E. 1968 The oscillations of a fluid droplet immersed in another fluid. J. Fluid Mech. 32, 417-435.

Natara.jan, R. \& Brown, R. A. 1987 The role of three-dimensional shapes in the break-up of charged drops. Proc. R. Soc. Lond. A 410, 209-227.

Prosperetti, A. 1977 Viscous effects on perturbed spherical flows. Q. Appl. Maths 34, $339-352$.

RaYleigh, J. W. S. 1879 On the capillary phenomena of jets. Proc. R. Soc. Lond. 29, 71-97.

ReId, W. H. 1960 The oscillations of a viscous liquid drop. Q. Appl. Maths 18, 86-89.

Ryskin, G. \& Leat, L. G. 1984 Numerical solutions of free-boundary problems in fluid mechanics. Part 3. Bubble deformation in an axisymmetric straining flow. J. Fluid Mech. 148, 37-43.

Subramanyam, S. V. 1969 A note on the damping and oscillations of a fluid drop moving in another fluid. J. Fluid Mech. 37, 715-725.

Tsamopoulos, J. A. \& Brown, R. A. 1983 Nonlinear oscillations of inviscid drops and bubbles. J. Fluid Mech. 127, 519-537.

Tsamopoulos, J. A. \& Brown, R. A. 1984 Resonant oscillations of inviscid charged drops. J. Fluid Mech. 147, 373-395.

VANDEN-Broeck, J.-M. \& Keller, J. B. 1980 Bubble or drop distortion in a straining flow in two dimensions. Phys. Fluids 23, 1491-1495. 